\title{
Crack nucleation in variational phase-field models of brittle fracture
}

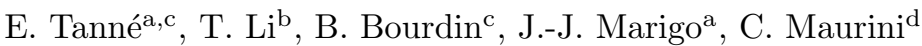 \\ ${ }^{a}$ Laboratoire de Mécanique des solides, École Polytechnique, Route de Saclay, 91120 Palaiseau, France \\ ${ }^{b}$ IMSIA (UMR EDF-CNRS-CEA-ENSTA ParisTech 9219), Universit Paris-Saclay, 91120 Palaiseau, France \\ ${ }^{c}$ Department of Mathematics and Center for Computation \& Technology, Louisiana State University, Baton Rouge \\ LA 70803, USA \\ ${ }^{d}$ Sorbonne Universités, UPMC Univ Paris 06, CNRS, UMR 7190, Institut Jean Le Rond d'Alembert, F-75005 Paris, \\ France
}

\begin{abstract}
Phase-field models, sometimes refered to as gradient damage or smeared crack models, are widely used methods for the numerical simulation of crack propagation in brittle materials. Theoretical results and numerical evidences show that they can predict the propagation of a pre-existing crack according to Griffith' criterion. For a one-dimensional problem, it has been shown that they can predict nucleation upon a critical stress, provided that regularization parameter be identified with the material's internal or characteristic length. In this article, we draw on numerical simulations to study crack nucleation in commonly encountered geometries for which closed-form solutions are not available. We use U- and $\mathrm{V}$-notches to show that the nucleation load varies smoothly from that predicted by a strength criterion to that of a toughness criterion, when the strength of the stress concentration or singularity varies. We present validation and verifications numerical simulations for both types of geometries. We consider the problem of an elliptic cavity in an infinite or elongated domain to show that variational phase field models properly account for structural and material size effects.

We conclude that variational phase-field models can accurately predict crack nucleation through energy minimization in a nonlinear damage model instead of introducing ad-hoc criteria.
\end{abstract}

Keywords: Phase-field models of fracture, crack nucleation, size effects in brittle materials, validation \& verification, gradient damage models, smeared crack models

\section{Introduction}

Despite its many successes, Griffith's theory of brittle fracture [51] and its heir, Linear Elastic Fracture Mechanics (LEFM), still faces many challenges. In order to identify crack path, additional branching criteria whose choice are still unsettled have to be considered. Accounting for scale effects in LEFM is also challenging, as illustrated by the following example: Consider a reference structure of unit size rescaled by a factor $L$. The critical loading at the onset of fracture scales then as $1 / \sqrt{L}$, leading to a infinite nucleation load as the structure size approaches 0 , which is inconsistent with experimental observation for small structures [10, 55, 30].

It is well accepted that this discrepency is due to the lack of a critical stress (or a critical lengthscale) in Griffith's theory. Yet, augmenting LEFM to account for a critical stress is also challenging. In essence, the idea of material strength is incompatible with the concept of elastic energy release rate near stress singularity, the pilar of Griffith-like theories as it would imply crack nucleation under a infinitesimal loading. Furthermore, a nucleation criterion based solely on pointwise maximum stress will be unable to handle crack formation in a body subject to a uniform stress distribution.

Many approaches have been proposed to provide models capable of addressing the aforementioned issues. Some propose to stray from Griffith fundamental hypotheses by incorporating cohesive fracture

Email addresses: erwan.tanne@gmail.com (E. Tanné), tianyikillua@gmail.com (T. Li), bourdin@lsu.edu (B. Bourdin), marigo@lms.polytechnique.fr (J.-J. Marigo), corrado.maurini@upmc.fr (C. Maurini) 
energies [76, 36, 35, 29] or material non-linearities [49]. Others have proposed dual-criteria involving both elastic energy release rate and material strength such as [61], for instance. Models based on the peridynamics theory [87] may present an alternative way to handle these issues, but to our knowlege, they are still falling short of providing robust quantitative predictions at the structural scale.

Francfort and Marigo [40] set to devise a formulation of brittle fracture based solely on Griffith's idea of competition between elastic and fracture energy, yet capable of handling the issues of crack path and crack nucleation. However, as already pointed-out in [40], their model inherits a fundamental limitation of the Griffith theory and LEFM: the lack of an internal length scale and of maximum allowable stresses.

Amongst many numerical methods originally devised for the numerical implementation of the Francfort-Marigo model [18, 74, 41, 84], Ambrosio-Tortorelli regularizations [5, 6], originally introduced in [20], have become ubiquitous. They are nowadays known as phase-field models of fracture, and share several common points with the approaches coming from Ginzburg-Landau models for phasetransition [57]. They have been applied to a wide variety of fracture problems including fracture of ferro-magnetic and piezo-electric materials [1, 90], thermal and drying cracks [68, 22], or hydraulic fracturing $[19,89,31,91]$ to name a few. They have been expended to account for dynamic effects, ductile behavior [2, 71, 3], cohesive effects [33, 32, 42], large deformations [4, 70, 14], or anisotropy [63], for instance.

Although phase-field models were originally conceived as approximations of Francfort and Marigo's variational approach to fracture in the vanishing limit of their regularization parameter, a growing body of literature is concerned with their links with gradient damage models [43, 66]. In this setting, the regularization parameter $\ell$ is kept fixed and interpreted as a material's internal length [80]. In particular, [78, 79] proposed an evolution principle for an Ambrosio-Tortorelli like energy based on irreversibility, stability and energy balance, where the regularization parameter $\ell$ is kept fixed and interpreted as a material's internal length. This approach, which we refer to as variational phase-field models, introduces a critical stress proportional to $1 / \sqrt{\ell}$. As observed in $[80,22,75]$, it can potentially reconcile stress and toughness criteria for crack nucleation, recover pertinent size effect at small and large length-scales, and provide a robust and relatively simple approach to model crack propagation in complex two- and three-dimensional settings. However, the few studies providing experimental verifications $[81,75,22]$ are still insufficient to fully support this conjecture.

The goal of this article is precisely to provide such evidences, focusing on nucleation and size-effects for mode-I cracks. We provide quantitative comparison of nucleation loads near stress concentrations and singularities with published experimental results for a range of materials. We show that variational phase-field models can reconcile strength and toughness thresholds and account for scale effect at the structural and the material length-scale. In passing, we leverage the predictive power of our approach to propose a new way to measure a material's tensile strength from the nucleation load of a crack near a stress concentration or a weak singularity. In this study, we focus solely on the identification of the critical stress at the first crack nucleation event and are not concerned by the post-critical fracture behavior.

The article is organized as follows: in Section 2, we introduce variational phase-field models and recall some of their properties. Section 3 focuses on the links between stress singularities and concentrations and crack nucleation in these models. We provide validation and verification results for nucleation induced by stress singularities using V-shaped notches, and concentrations using U-notches. Section 4 is concerned with shape and size effects. We investigate the role of the internal length on nucleation near a defect, focusing on an elliptical cavity and a mode-I crack, and discussing scale effects at the material and structural length scales. Conclusions are finally drawn in Section 5.

\section{Variational phase-field models}

We start by recalling some important properties of variational phase-field models, focussing first on their construction as approximation method of Francfort and Marigo's variational approach to fracture, then on their alternative formulation and interpretation as gradient-damage models. 


\subsection{Regularization of the Francfort-Marigo fracture energy}

Consider a perfectly brittle material with Hooke's law $A$ and critical elastic energy release rate $G_{c}$ occupying a region $\Omega \subset \mathbb{R}^{n}$, subject to a time dependent boundary displacement $\bar{u}$ on a part $\partial_{D} \Omega$ of its boundary and stress free on the remainder $\partial_{N} \Omega$. In the variational approach to fracture, the quasi-static equilibrium displacement $u_{i}$ and crack set $\Gamma_{i}$ at a given discrete time step $t_{i}$ are given by the minimization problem (see also [21])

$$
\left(u_{i}, \Gamma_{i}\right)=\underset{\substack{u=\bar{u}_{i} \text { on } \partial_{D} \Omega \\ \Gamma \supset \Gamma_{i-1}}}{\operatorname{argmin}} \mathcal{E}(u, \Gamma):=\int_{\Omega \backslash \Gamma} \frac{1}{2} A \mathrm{e}(u) \cdot \mathrm{e}(u) d x+G_{c} \mathcal{H}^{n-1}\left(\Gamma \cap \bar{\Omega} \backslash \partial_{N} \Omega\right),
$$

where $\mathcal{H}^{n-1}(\Gamma)$ denotes the Hausdorff $n$-1-dimensional measure of the unknown crack $\Gamma$, i.e. its aggregate length in two dimensions or surface area in three dimensions, and e $(u):=\frac{1}{2}\left(\nabla u+\nabla^{T} u\right)$ denotes the symmetrized gradient of $u$.

Because in (1) the crack geometry $\Gamma$ is unknown, special numerical methods had to be crafted. Various approaches based for instance on adaptive or discontinuous finite elements were introduced [18, 46, 41]. Variational phase-field methods, take their roots in Ambrosio and Tortorelli's regularization of the Mumford-Shah problem in image processing [5,6], adapted to brittle fracture in [20]. In this framework, a regularized energy $\mathcal{E}_{\ell}$ depending on a regularization length $\ell>0$ and a "phase-field" variable $\alpha$ taking its values in $[0,1]$ is introduced. A broad class of such functionals was introduced in [23]. They are

$$
\mathcal{E}_{\ell}(u, \alpha)=\int_{\Omega} \frac{a(\alpha)+\eta_{\ell}}{2} A \mathrm{e}(u) \cdot \mathrm{e}(u) d x+\frac{G_{c}}{4 c_{w}} \int_{\Omega} \frac{w(\alpha)}{\ell}+\ell|\nabla \alpha|^{2} d x
$$

where $a$ and $w$ are continuous monotonic functions such that $a(0)=1, a(1)=0, w(0)=0$, and $w(1)=1, \eta_{\ell}=o(\ell)$, and $c_{w}:=\int_{0}^{1} \sqrt{w(s)} d s$ is a normalization parameter. The approximation of $\mathcal{E}$ by $\mathcal{E}_{\ell}$ takes place with the framework of $\Gamma$-convergence (see [34, 24] for instance). More precisely, if $\mathcal{E}_{\ell} \Gamma$-converges to $\mathcal{E}$, then the global minimizers of $\mathcal{E}_{\ell}$ converge to that of $\mathcal{E}$. The $\Gamma$-convergence of a broad class of energies, including the ones above was achieved with various degrees of refinement going from static scalar elasticity to time discrete and time continuous quasi-static evolution linearized elasticity, and their finite element discretization [12, 16, 23, 46, 27, 28, 45, 25, 26, 56].

Throughout this article, we focus on two specific models:

$$
\mathcal{E}_{\ell}(u, \alpha)=\int_{\Omega} \frac{(1-\alpha)^{2}+\eta_{\ell}}{2} A \mathrm{e}(u) \cdot \mathrm{e}(u) d x+\frac{G_{c}}{2} \int_{\Omega} \frac{\alpha^{2}}{\ell}+\ell|\nabla \alpha|^{2} d x,
$$

introduced in [6] for the Mumford-Shah problem and in [20] for brittle fracture, and

$$
\mathcal{E}_{\ell}(u, \alpha)=\int_{\Omega} \frac{(1-\alpha)^{2}+\eta_{\ell}}{2} A \mathrm{e}(u) \cdot \mathrm{e}(u) d x+\frac{3 G_{c}}{8} \int_{\Omega} \frac{\alpha}{\ell}+\ell|\nabla \alpha|^{2} d x
$$

used in [22].

The "surfing" problem introduced in [53] consists in applying a translating boundary displacement on $\Omega$ given by $\bar{u}(x, y)=\bar{u}_{I}(x-V t, y)$, where $\bar{u}_{I}$ denotes the asymptotic far-field displacement field associated with a mode-I crack along the $x$-axis with tip at $(0,0), V$ is a prescribed loading "velocity", and $t$ a loading parameter ("time"). Figure 1(left) shows the outcome of a surfing experiment on a rectangular domain $\Omega=[0,5] \times\left[-\frac{1}{2}, \frac{1}{2}\right]$ with an initial crack $\Gamma_{0}=\left[0, l_{0}\right] \times\{0\}$ for several values of $\ell$. The $\mathrm{AT}_{1}$ model is used, assuming plane stress conditions, and the mesh size $h$ is adjusted so that $\ell / h=5$, keeping the "effective" numerical toughness $G_{\text {eff }}$, (see [21]) fixed. The Poisson ratio is $\nu=0.3$, the Young's modulus is $E=1$, the fracture toughness is $G_{c}=1.5$, and the loading rate $V=4$. As expected, after a transition stage, the crack length depends linearly on the loading parameter with slope $3.99,4.00$ and 4.01 for values of $\ell=$ of $0.1,0.05$ and 0.025 respectively. The elastic energy release rate $G$, computed using the $G_{\theta}$ method $[37,86,65]$ is very close to $G_{\text {eff. }}$ Even though $\Gamma$-convergence only mandates that the elastic energy release rate in the regularized energy converges to that of Griffith as 

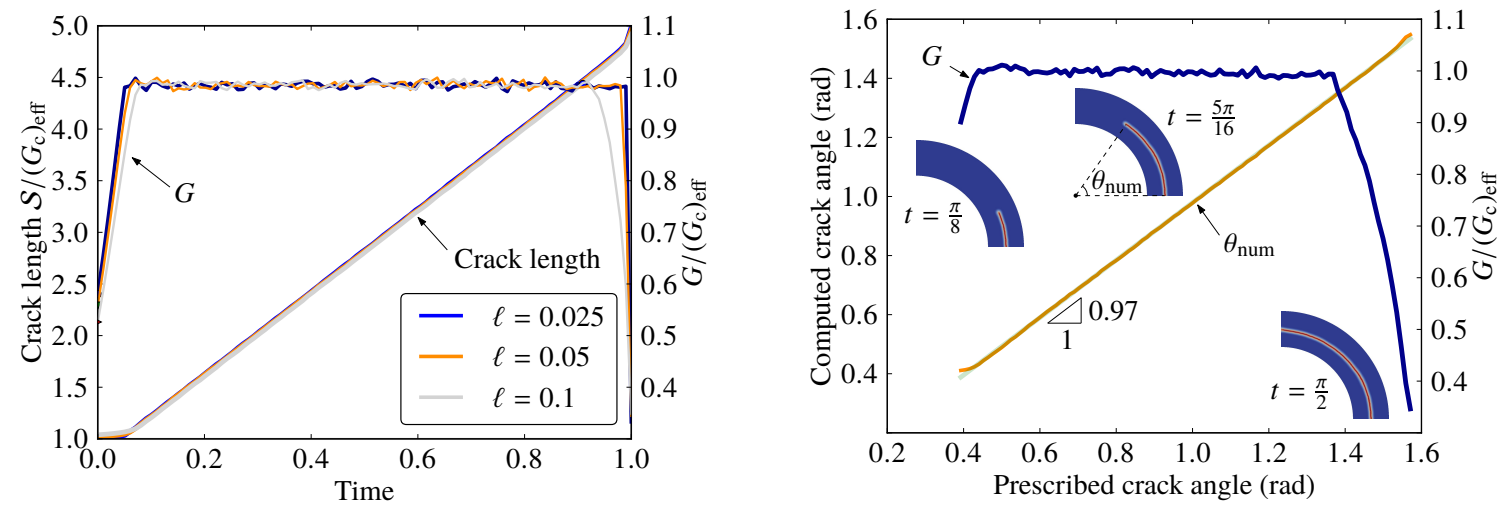

Figure 1: Mode-I "surfing" experiment along straight (left) and circular (right) paths. Dependence of the crack length and elastic energy release rate on the loading parameter for multiple values of $\ell$.

$\ell \rightarrow 0$, we observe that as long as $\ell$ is "compatible" with the discretization size and domain geometry, its influence on crack propagation is insignificant. Similar observations were reported in [58, 93, 81].

Figure 1(right) repeats the same experiment for a curve propagating along a circular path. Here, the boundary displacement is given by Muskhelishvili's exact solution for a crack propagating in modeI along a circular path [73]. The Young's modulus, fracture toughness, loading rate are set to 1. Again, we see that even for a fixed regularization length, the crack obeys Griffith's criterion.

When crack nucleation is involved, the picture is considerably different. Consider a one-dimensional domain of length $L$, fixed at one end and submitted to an applied displacement $\bar{u}=e L$ at the other end. For the lack of an elastic singularity, LEFM is incapable of predicting crack nucleation here, and predicts a structure capable of supporting arbitrarily large loads without failing. A quick calculation shows that the global minimizer of (1) corresponds to an uncracked elastic solution if $e<e_{c}:=\sqrt{\frac{2 G_{c}}{E L}}$, while at $e=e_{c}$, a single crack nucleates at an arbitrary location (see [40, 21]). The failure stress is $\sigma_{c}=\sqrt{2 G_{c} E / L}$, which is consistent with the scaling law $\sigma_{c}=\mathcal{O}(1 / \sqrt{L})$ mentioned in the introduction. The uncracked configuration is always a stable local minimizer of (1), so that if local minimization of (1) is considered, nucleation never takes place. Just as before, one can argue that for the lack of a critical stress, an evolution governed by the generalized Griffith energy (1) does not properly account for nucleation and scaling laws.

When performing global minimization of (2) using the backtracking algorithm of [17] for instance, a single crack nucleates at an $\ell$-dependent load. As predicted by the $\Gamma$-convergence of $\mathcal{E}_{\ell}$ to $\mathcal{E}$, the critical stress at nucleation converges to $\sqrt{2 G_{c} E / L}$ as $\ell \rightarrow 0$. Local minimization of (2) using the alternate minimizations algorithm of [20], or presumedly any gradient-based monotonically decreasing scheme, leads to the nucleation of a single crack at a critical load $e_{c}$, associated with a critical stress $\sigma_{c}=\mathcal{O}\left(\sqrt{G_{c} E / \ell}\right)$. In the limit of vanishing $\ell$, local and global minimization of (2) inherit therefore the weaknesses of Griffith-like theories when dealing with scaling properties and crack nucleation.

\subsection{Variational phase-field models as gradient damage models}

More recent works have seek to leverage the link between $\sigma_{c}$ and $\ell$. Ambrosio-Tortorelli functionals are then seen as the free energy of a gradient damage model $[43,66,13,78,79]$ where $\alpha$ plays the role of a scalar damage field. In [80], a throrough investigation of a one-dimensional tension problem led to interpreting $\ell$ as a material's internal or characteristic length linked to a material's tensile strength. An overview of this latter approach, which is the one adopted in the rest of this work, is given below.

In all that follows, we focus on a time-discrete evolution but refer the reader to $[78,79,67]$ for a time-continuous formulation which can be justified within the framework of generalized standard materials [52] and rate-independent processes [72]. At any time step $i>1$, the sets of admissible 
displacement and damage fields $\mathcal{C}_{i}$ and $\mathcal{D}_{i}$, equipped with their natural $H^{1}$ norm, are

$$
\begin{aligned}
\mathcal{C}_{i} & =\left\{u \in H^{1}(\Omega): u=\bar{u}_{i} \text { on } \partial_{D} \Omega\right\}, \\
\mathcal{D}_{i} & =\left\{\beta \in H^{1}(\Omega): \alpha_{i-1}(x) \leq \beta(x) \leq 1, \quad \forall x \in \Omega\right\},
\end{aligned}
$$

where the constraint $\alpha_{i-1}(x) \leq \beta(x) \leq 1$ in the definition of $\mathcal{D}_{i}$ mandates that the damage be an increasing function of time, accounting for the irreversible nature of the damage process. The damage and displacement fields $\left(u_{i}, \alpha_{i}\right)$ are then local minimizers of the energy $\mathcal{E}_{\ell}$, i.e. there exists $h_{i}>0$ such that

$$
\forall(v, \beta) \in \mathcal{C}_{i} \times \mathcal{D}_{i} \text { such that }\left\|(v, \beta)-\left(u_{i}, \alpha_{i}\right)\right\| \leq h_{i}, \mathcal{E}_{\ell}\left(u_{i}, \alpha_{i}\right) \leq \mathcal{E}_{\ell}(v, \beta),
$$

where $\|\cdot\|$ denotes the natural $H^{1}$ norm of $\mathcal{C}_{i} \times \mathcal{D}_{i}$.

We briefly summarize the solution of the uniaxial tension of a homogeneous bar [77, 80], referring the reader to the recent review [67] for further details: As one increases the applied strain, the damage field remains 0 and the stress field constant until it reaches the elastic limit

$$
\sigma_{e}=\sqrt{\frac{G_{c} E}{c_{w} \ell}} \sqrt{\frac{w^{\prime}(0)}{2 s^{\prime}(0)}} .
$$

where $E$ is the Young modulus of the undamaged material, and $s(\alpha)=1 / a(\alpha)$. If the applied displacement is increased further, the damage field increases but remains spatially constant. Stress hardening is observed until peak stress $\sigma_{c}$, followed by stress softening. A stability analysis shows that for long enough domains (i.e. when $L \gg \ell$ ), the homogeneous solution is never stable in the stress softening phase, and that a snap-back to a fully localized solution such that $\max _{x \in(0, L)} \alpha(x)=1$ is observed. The profile of the localized solution and the width $D$ of the localization can be derived explicitly from the functions $a$ and $w$. With the choice of normalization of (2), the surface energy associated to the fully localized solution is exactly $G_{c}$ and its elastic energy is 0 , so that the overall response of the bar is that of a brittle material with toughness $G_{c}$ and strength $\sigma_{c}$.

Knowing the material's toughness $G_{c}$ and the Young's modulus $E$, one can then adjust $\ell$ in such a way that the peak stress $\sigma_{c}$ matches the nominal material's strength. Let us denote by

$$
\ell_{c h}=\frac{G_{c} E^{\prime}}{\sigma_{c}^{2}}=\frac{K_{I c}^{2}}{\sigma_{c}^{2}}
$$

the classical material's characteristic length(see [82,39], for instance), where $E^{\prime}=E$ in three dimension and in plane stress, or $E^{\prime}=\frac{E}{1-\nu^{2}}$ in plane strain. The identification above gives

$$
\ell_{1}:=\frac{3}{8} \ell_{c h} ; \quad \ell_{2}:=\frac{27}{256} \ell_{c h},
$$

for the $\mathrm{AT}_{1}$ and $\mathrm{AT}_{2}$ models, respectively.

Table 1 summarizes the specific properties of the $\mathrm{AT}_{1}$ and $\mathrm{AT}_{2}$ models. The $\mathrm{AT}_{1}$ model has some key conceptual and practical advantages over the $\mathrm{AT}_{2}$ model used in previous works, which were leveraged in [22] for instance: It has a non-zero elastic limit, preventing diffuse damage at small loading. The length localization band $D$ is finite so that equivalence with Griffith energy is obtained even for a finite value of $\ell$, and not only in the limit of $\ell \rightarrow 0$, as predicted by $\Gamma$-convergence [86]. By remaining quadratic in the $\alpha$ and $u$ variables, its numerical implementation using alternate minimizations originally introduced in [20] is very efficient.

In all the numerical simulations presented below, the energy (2) is discretized using linear Lagrange elements, and minimization performed by alternating minimization with respect to $u$ and $\alpha$. Minimization with respect to $u$ is a simple linear problem solved using preconditioned gradient conjugated while constrained minimization with respect to $\alpha$ is reformulated as a variational inequality and implemented using the variational inequality solvers provided by PETSc $[9,8,7]$. All computations were performed using the open source implementations models mef $90^{1}$ and gradient-damage ${ }^{2}$.

\footnotetext{
1 available at https://www.bitbucket.org/bourdin/mef90-sieve

2 available at https://bitbucket.org/cmaurini/gradient-damage
} 


\begin{tabular}{cccccccc}
\hline model & $w(\alpha)$ & $a(\alpha)$ & $c_{w}$ & $\sigma_{e}$ & $\sigma_{c}$ & $D$ & $\ell_{c h}$ \\
\hline $\mathrm{AT}_{1}$ & $\alpha$ & $(1-\alpha)^{2}$ & $\frac{2}{3}$ & $\sqrt{\frac{3 G_{c} E^{\prime}}{8 \ell}}$ & $\sqrt{\frac{3 G_{c} E^{\prime}}{8 \ell}}$ & $4 \ell$ & $\frac{8}{3} \ell$ \\
$\mathrm{AT}_{2}$ & $\alpha^{2}$ & $(1-\alpha)^{2}$ & $\frac{1}{2}$ & 0 & $\frac{3}{16} \sqrt{\frac{3 G_{c} E^{\prime}}{\ell}}$ & $\infty$ & $\frac{256}{27} \ell$ \\
\hline
\end{tabular}

Table 1: Properties of the gradient damage models considered in this work: the elastic limit $\sigma_{e}$, the material strength $\sigma_{c}$, the width of the damage band $D$, and the conventional material length $\ell_{c h}$ defined in (5). We use the classical convention $E^{\prime}=E$ in three dimension and in plane stress, and $E^{\prime}=\frac{E}{1-\nu^{2}}$ in plane strain.

\section{Effect of stress concentrations}

The discussion above suggests that variational phase-field models, as presented in Section 2.2, can account for strength and toughness criteria simultaneously, on an idealized geometry. We propose to investigate this claim further by focussing on more general geometries, a V-shaped notch to illustrate nucleation near stress singularities and a U-shaped notch for stress concentrations. There is a wealth of experimental literature on crack initiation in such geometries using three-point bending (TPB), four-point bending (FPB), single or double edge notch tension (SENT and DENT) allowing us to provide qualitative validation and verification simulations of the critical load at nucleation.

\subsection{Initiation near a weak stress singularity: the V-notch}

Consider a $\mathrm{V}$-shaped notch in a linear elastic isotropic homogeneous material. Let $(r, \theta)$ be the polar coordinate system emanating from the notch tip with $\theta=0$ corresponding to the notch symmetry axis, shown on Figure 2(left). Assuming that the notch lips $\Gamma^{+} \cup \Gamma^{-}$are stress free, the mode-I component of the singular part of the stress field in plane strain is given in [62]:

$$
\begin{aligned}
\sigma_{\theta \theta} & =k r^{\lambda-1} F(\theta), \\
\sigma_{r r} & =k r^{\lambda-1} \frac{F^{\prime \prime}(\theta)+(\lambda+1) F(\theta)}{\lambda(\lambda+1)}, \\
\sigma_{r \theta} & =-k r^{\lambda-1} \frac{F^{\prime}(\theta)}{(\lambda+1)},
\end{aligned}
$$

where

$$
F(\theta)=(2 \pi)^{\lambda-1} \frac{\cos ((1+\lambda) \theta)-f(\lambda, \bar{\omega}) \cos ((1-\lambda) \theta)}{1-f(\lambda, \bar{\omega})},
$$

and

$$
f(\lambda, \bar{\omega})=\frac{(1+\lambda) \sin ((1+\lambda)(\pi-\bar{\omega}))}{(1-\lambda) \sin ((1-\lambda)(\pi-\bar{\omega}))}
$$

and the exponent of the singularity $\lambda \in[1 / 2,1]$, see Figure 2(right), solves

$$
\sin (2 \lambda(\pi-\bar{\omega}))+\lambda \sin (2(\pi-\bar{\omega}))=0 .
$$

From (7), it is natural to define a generalized stress intensity factor

$$
k=\left.\frac{\sigma_{\theta \theta}}{(2 \pi r)^{\lambda-1}}\right|_{\theta=0} .
$$

Note that this definition differs from the one often encountered in the literature by a factor $(2 \pi)^{\lambda-1}$, so that when $\omega=0$ (i.e. when the notch degenerates into a crack), $k$ corresponds to the mode-I stress 

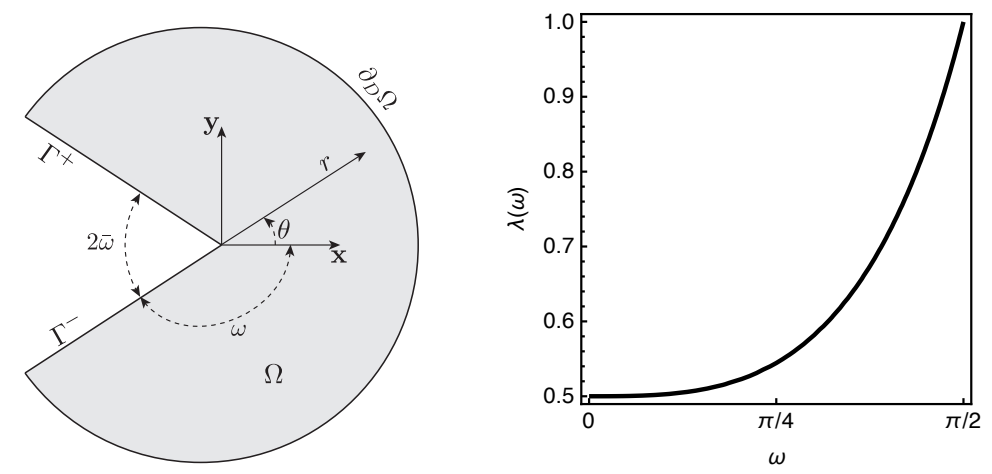

Figure 2: Pac-man geometry for the study of the crack nucleation at a notch. Left: sketch of the domain and notation. Right: relation between the exponent of the singularity $\lambda$ and the notch opening angle $\bar{\omega}$ determined by the solution of equation (10). For any opening angle $\bar{\omega}$ we apply on $\partial_{D} \Omega$ the displacement boundary condition obtained by evaluating on $\partial_{D} \Omega$ the asymptotic displacement (12) with $\lambda=\lambda(\omega)$.

intensity factor whereas when $\omega=\pi / 2, k$ is the tangential stress, and that the physical dimension of $[k] \equiv \mathrm{N} / \mathrm{m}^{-\lambda-1}$ is not a constant but depends on the singularity power $\lambda$.

If $\bar{\omega}<\pi / 2$ (i.e. $\omega>\pi / 2$ ), the stress field is singular at the notch tip so that a nucleation criterion based on maximum pointwise stress will predict crack nucleation for any arbitrary small loading. Yet, as long as $\bar{\omega}>0(\omega<\pi)$, the exponent of the singularity is sub-critical in the sense of Griffith, so that LEFM forbids crack nucleation, regardless of the magnitude of the loading.

\subsubsection{The mode-I Pac-Man test}

Consider a Pac-Man-shaped ${ }^{3}$ domain with radius $L \gg \ell$ and notch angle $\bar{\omega}$ as in Figure 2(left). In linear elasticity, a displacement field associated with the stress field (7) is

$$
\begin{aligned}
& \bar{u}_{r}=\frac{r^{\lambda}}{E} \frac{\left(1-\nu^{2}\right) F^{\prime \prime}(\theta)+(\lambda+1)\left[1-\nu \lambda-\nu^{2}(\lambda+1)\right] F(\theta)}{\lambda^{2}(\lambda+1)} \\
& \bar{u}_{\theta}=\frac{r^{\lambda}}{E} \frac{\left(1-\nu^{2}\right) F^{\prime \prime \prime}(\theta)+\left[2(1+\nu) \lambda^{2}+(\lambda+1)\left(1-\nu \lambda-\nu^{2}(\lambda+1)\right] F^{\prime}(\theta)\right.}{\lambda^{2}\left(1-\lambda^{2}\right)} .
\end{aligned}
$$

In the mode-I Pac-Man test, we apply a boundary displacement of the form $t \bar{u}$, where $t$ is a monotonically increasing loading parameter on the outer edge of the domain $\partial_{D} \Omega$.

We performed series of numerical simulations varying the notch angle $\bar{\omega}$ and regularization parameter $\ell$ for the $\mathrm{AT}_{1}$ and $\mathrm{AT}_{2}$ models. Up to a rescaling and without loss of generality, it is always possible to assume that $E=1$ and $G_{c}=1$. The Poisson ratio was set to $\nu=0.3$. We either prescribed the value of the damage field on $\Gamma^{+} \cup \Gamma^{-}$to 1 (we refer this to as "damaged notch conditions") or let it free ("undamaged notch conditions"). The mesh size was kept at a fixed ratio of the internal length $h=\ell / 5$.

For "small" enough loadings, we observe an elastic or nearly elastic phase during which the damage field remains 0 or near 0 away from an area of radius $o(\ell)$ near the notch tip. Then, for some loading $t=t_{c}$, we observed the initiation of a "large" add-crack associated with a sudden jump of the elastic and surface energy. Figure 3 shows a typical mesh, the damage field immediately before and after nucleation of a macroscopic crack and the energetic signature of the nucleation event.

Figure 4 shows that up to the critical loading, the generalized stress intensity factor can be accurately recovered by averaging $\sigma_{\theta \theta}(r, 0) /(2 \pi r)^{\lambda-1}$ along the symmetry axis of the domain, provided that the region $r \leq 2 \ell$ be excluded.

Figure 5(left) shows the influence of the internal length on the critical generalized stress intensity factor for a sharp notch $\left(\bar{\omega}=0.18^{\circ}\right)$ for the $\mathrm{AT}_{1}$ and $\mathrm{AT}_{2}$ models, using damaged and undamaged notch

\footnotetext{
${ }^{3}$ https://en.wikipedia.org/wiki/Pac-Man
} 

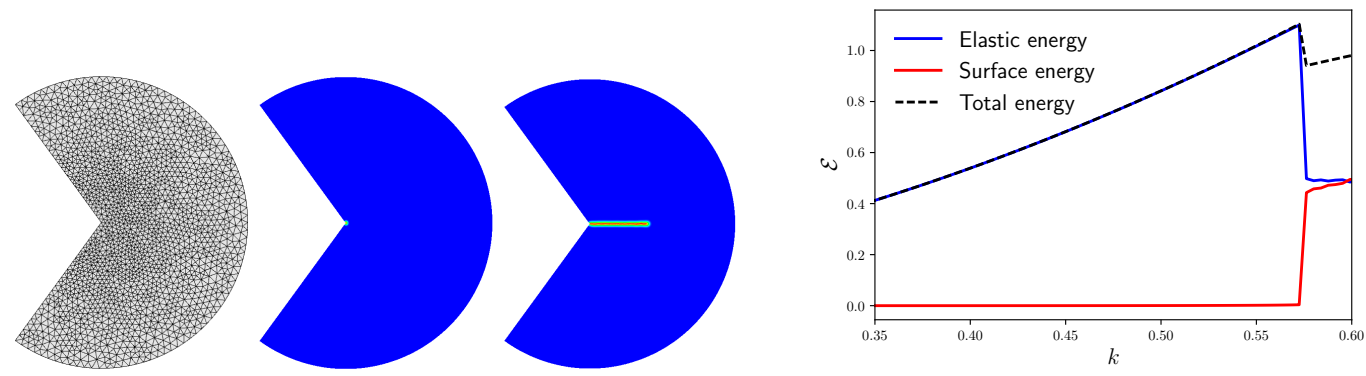

Figure 3: Pac-Man test with the $\mathrm{AT}_{1}$ model, $\ell=0.015, \omega=0.7 \pi$, and $\nu=0.3$. From left to right: typical mesh (with element size ten times larger than that in typical simulation for illustration purpose), damage field immediately before and after the nucleation of a crack, and plot of the energies versus the loading parameter $k$. Note the small damaged zone ahead of the notch tip before crack nucleation, and the energetic signature of a nucleation event.
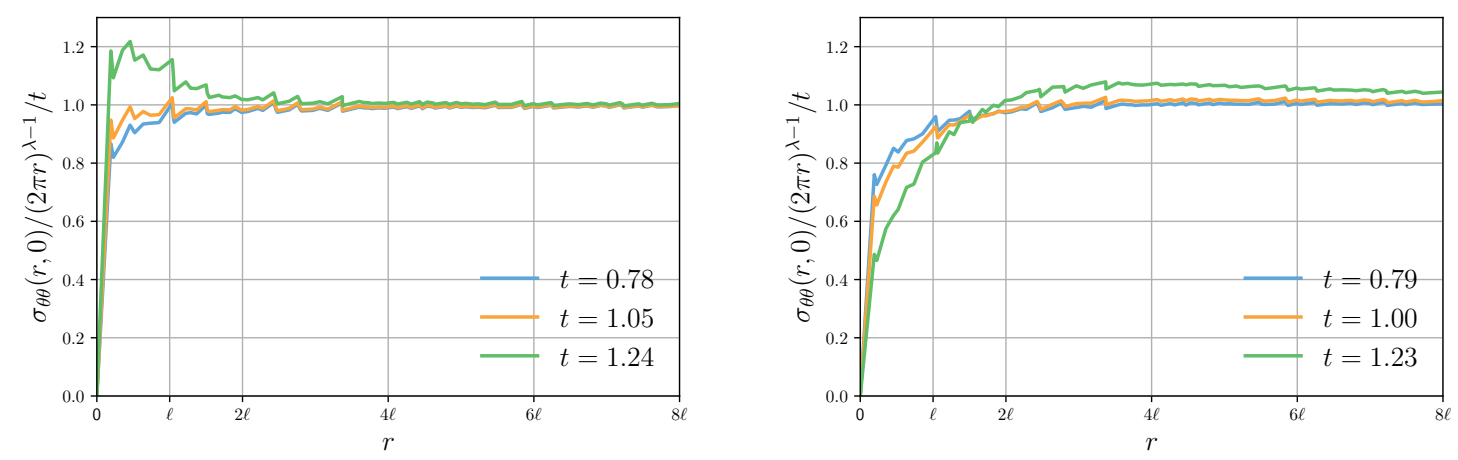

Figure 4: Identification of the generalized stress intensity factor: $\frac{\sigma_{\theta \theta}(r, 0)}{(2 \pi r)^{\lambda-1}}$ along the domain symmetry axis for the $\mathrm{AT}_{1}$ (left) and $\mathrm{AT}_{2}$ (right) models with undamaged notch conditions, and sub-critical loadings. The notch aperture is $\bar{\omega}=\pi / 10$

boundary conditions on the damage field. In this case, the generalized stress intensity factor coincides with the standard mode-I stress intensity factor $K_{I c}$. As suggested by the surfing experiment in the introduction, the internal length $\ell$ also has a very minor influence on the critical load $t:=k_{c}^{A T} \simeq$ $K_{I c}=\sqrt{G_{c} E^{\prime}}$. As reported previously in [58] for instance, undamaged notch conditions lead to overestimating the critical load. We speculate that this is because with undamaged notch condition, the energy barrier associated with bifurcation from an undamaged (or partially damaged) state to a fully localized state needs to be overcome. As expected, this energy barrier is larger for the $\mathrm{AT}_{1}$ model than for the $\mathrm{AT}_{2}$ model for which large damaged areas ahead of the notch tip are observed.

For flat notches $\left(2 \bar{\omega}=179.64^{\circ}\right)$ as shown in Figure 5 (right), the generalized stress intensity factor $k$ takes the dimension of a stress, and crack nucleation is observed when $k_{c}$ reaches the $\ell$-dependent value $\sigma_{c}$ given in Table 1, i.e. when $\left.\sigma_{\theta \theta}\right|_{\theta=0}=\sigma_{c}$, as in the uniaxial tension problem. In this case the type of damage boundary condition on the notch seems to have little influence.

For intermediate values of $\bar{\omega}$, we observe in Figure 6 that the critical generalized stress intensity factor varies smoothly and monotonically between its extreme values and remains very close to $K_{I c}$ for opening angles as high as $30^{\circ}$ which justifies the common numerical practice of replacing initial cracks with slightly open sharp notches and damaged notch boundary conditions.

\subsubsection{Validation}

For intermediate values $0<2 \bar{\omega}<\pi$, verification is rendered more difficult by the fact that various models predict different nucleation loads. Instead, we focus on validation against experiments from the literature based on measurements of the generalized stress intensity factor at a V-shaped notch. 

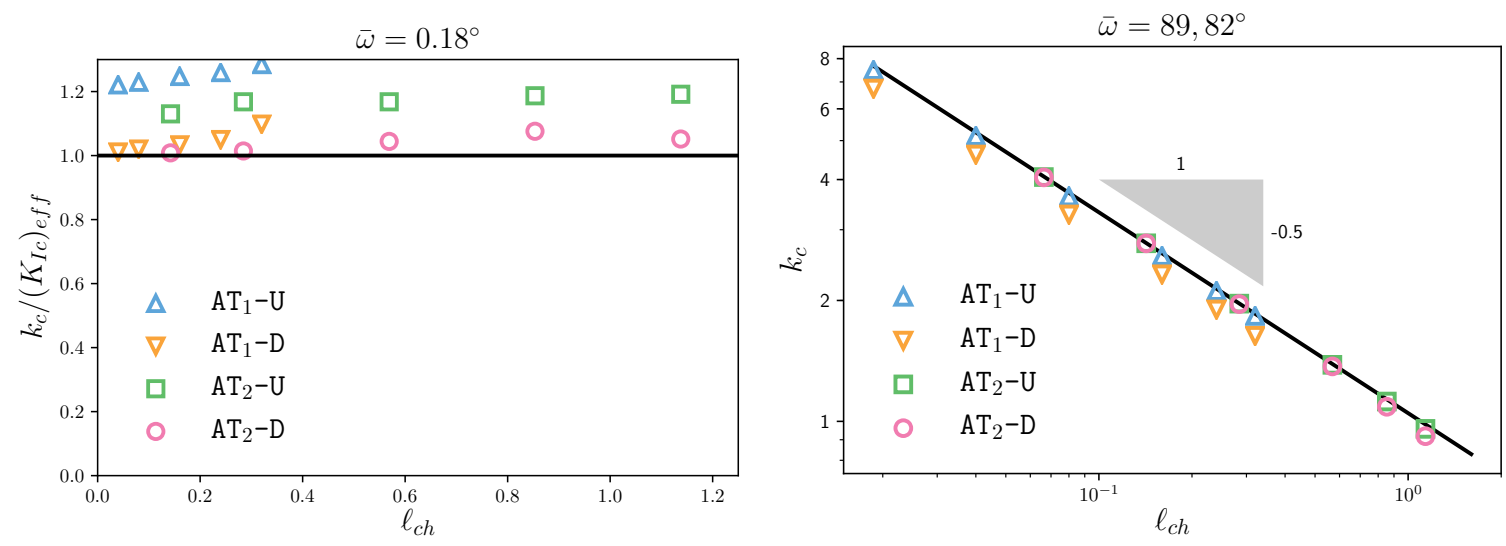

Figure 5: Critical generalized critical stress intensity factor at crack nucleation as a function of the internal length for $\bar{\omega} \simeq 0$ (left) and $\omega \simeq \pi / 2$ (right). $\mathrm{AT}_{1}-\mathrm{U}, \mathrm{AT}_{1}-\mathrm{D}, \mathrm{AT}_{2}-\mathrm{U}$, and $\mathrm{AT}_{2}-\mathrm{D}$ refer respectively to computations using the $\mathrm{AT}_{1}$ model with damaged notch and undamaged notch boundary conditions, and the $\mathrm{AT}_{2}$ model with damaged notch and undamaged notch boundary conditions.

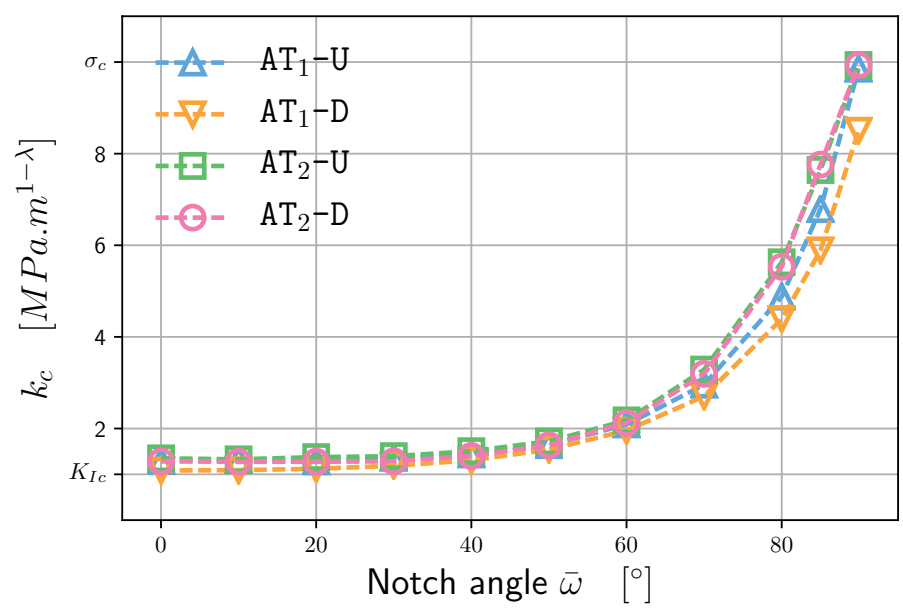

Figure 6: Critical generalized stress intensity factor $k$ for crack nucleation at a notch as a function of the notch opening angle $\bar{\omega}$. Results for the $\mathrm{AT}_{1}$ and $\mathrm{AT}_{2}$ models with damaged $-\mathrm{D}$ and undamaged $-\mathrm{U}$ notch lips conditions. The results are obtained with numerical simulations on the Pac-Man geometry with $\left(K_{i c}\right)_{\text {eff }}=1$ and $\sigma_{c}=10$ under plane-strain conditions with a unit Young's modulus and a Poisson ratio $\nu=0.3$.

Data from single edge notch tension (SENT) test of soft annealed tool steel, (AISI O1 at $-50^{\circ} \mathrm{C}$ ) [88], four point bending (FPB) experiments of Divinycell@ H80, H100, H130, and H200 PVC foams) [50], and double edge notch tension (DENT) experiments of poly methyl methacrylate (PMMA) and duraluminium [85], were compiled in [47]. We performed a series of numerical simulations of Pac-Man tests using the material properties reported in [47] and listed in Table 2. In all cases, the internal length $\ell$ was computed using (6).

Figures 7 and 8 compare the critical generalized stress intensity factor from our numerical simulations with experimental values reported in the literature for $\mathrm{V}$-notch with varying aperture. The definition (11) for $k$ is used. In each case, we observe a remarkable agreement for the entire range of notch openings.

The numerical values of the critical generalized stress intensity factors for the $\mathrm{AT}_{1}$ models and the experiments from the literature are included in Tables A.4, A.5, A.6, and A.7 using the convention of (11) for $k$. As suggested by Figure 5 and reported in the literature [see 58], nucleation is best captured if damaged notch boundary conditions are used for sharp notches and undamaged notch 


\begin{tabular}{llllll}
\hline Material & $E[\mathrm{MPa}]$ & $\nu$ & $K_{I c}[\mathrm{MPa} \sqrt{\mathrm{m}}]$ & $\sigma_{c}[\mathrm{MPa}]$ & source \\
\hline $\mathrm{Al}_{2} \mathrm{O}_{3}-7 \% \mathrm{ZrO}_{2}$ & 350,000 & 0.24 & 4.1 & 290 & {$[92]$} \\
\hline PMMA & 2,300 & 0.36 & 1.03 & 124 & {$[38,92]$} \\
\hline Plexiglass & 3,000 & 0.36 & 1.86 & 104.9 & {$[85]$} \\
\hline PVC H80 & 85 & 0.32 & 0.32 & 2.51 & {$[47,50]$} \\
PVC H100 & 125 & 0.32 & 0.26 & 4.02 & {$[47,50]$} \\
PVC H130 & 175 & 0.32 & 0.34 & 5.70 & {$[47,50]$} \\
PVC H200 & 310 & 0.32 & 0.57 & 9.38 & {$[47,50]$} \\
\hline Steel & 205,000 & 0.3 & 52 & 1170 & {$[47,88]$} \\
\hline Duraluminium & 70,000 & 0.3 & 50.6 & 705 & {$[85]$} \\
\hline
\end{tabular}

Table 2: Material properties used in the numerical simulations as given in the literature
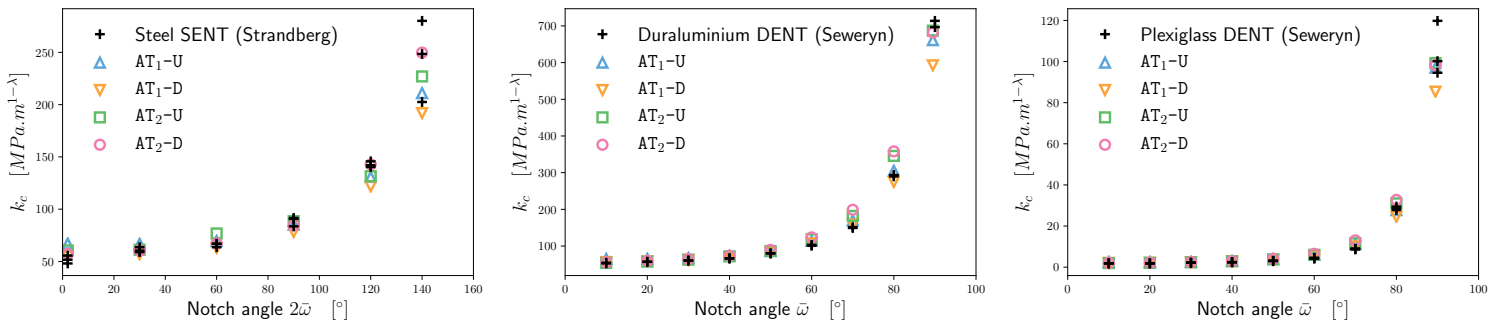

Figure 7: Critical generalized stress intensity factor $k_{c}$ vs notch angle. Comparison between numerical simulations with the $\mathrm{AT}_{1}$ and $\mathrm{AT}_{2}$ models and damaged and undamaged boundary conditions on the notch edges with experiments in steel from [88] (left), and Duraluminium (middle) and PMMA (right) from [85].
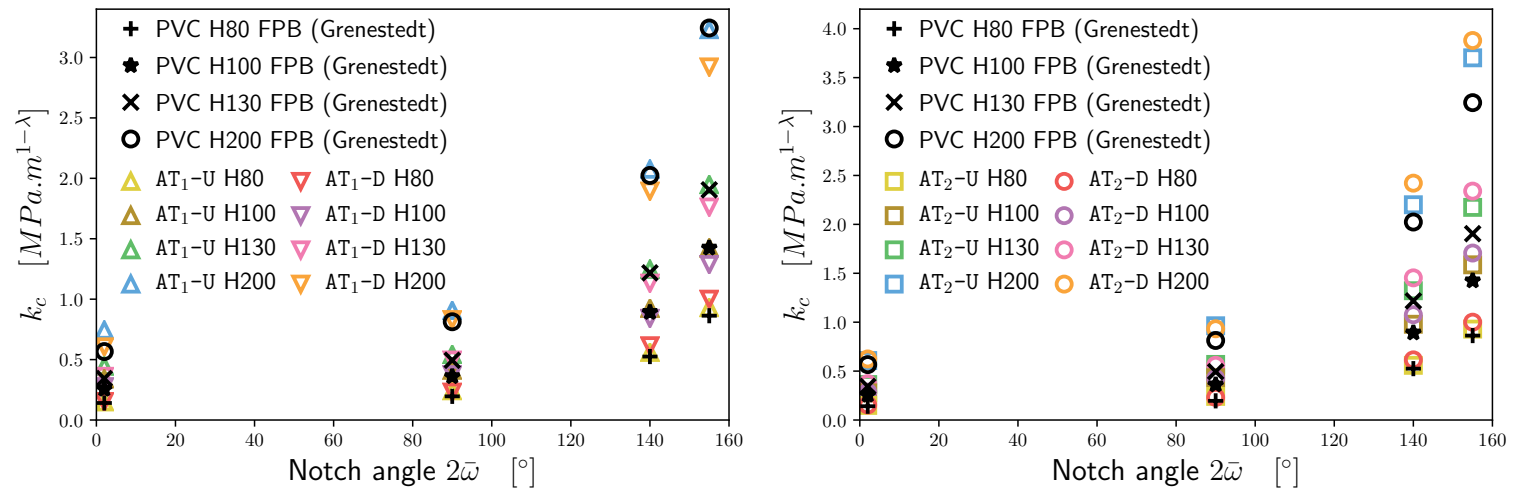

Figure 8: Critical generalized stress intensity factor $k_{c}$ vs notch angle and depth in PVC foam samples from [50]. Numerical simulations with the $\mathrm{AT}_{1}$ model with damaged and undamaged notch conditions (left), and $\mathrm{AT}_{2}$ model with damaged and undamaged notch conditions (right).

conditions for flat ones.

These examples strongly suggest that variational phase-field models of fracture are capable of predicting mode-I nucleation in stress and toughness dominated situations, as seen above, but also in the intermediate cases. Conceptually, toughness and strength (or equivalently internal length) could be measured by matching generalized stress intensity factors in experiments and simulations. When doing so, however, extreme care has to be exerted in order to ensure that the structural geometry has no impact on the measured generalized stress. Similar experiments were performed in [38, 92] for three and four point bending experiments on PMMA and Aluminium oxyde-Zirconia ceramics samples. While the authors kept the notch angle fixed, they performed three and four point bending experiments or varied the relative depth of the notch as a fraction of the sample height (see Figure 9). 

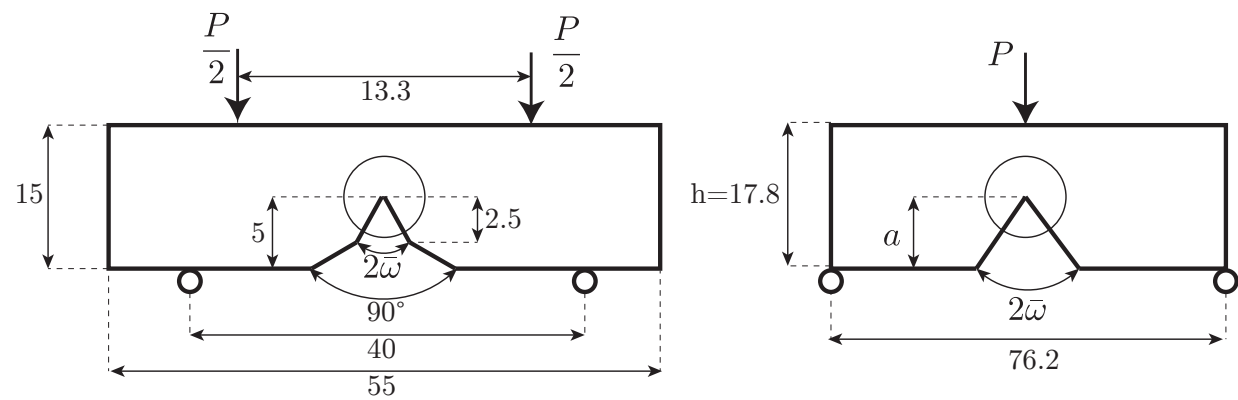

Figure 9: Schematic of the geometry and loading in the four point bending experiments of [92] (left) and three point bending experiments of [38] (right). The geometry of the three point bending experiment of [92] is identical to that of their four point bending, up to the location of the loading devices.

Figure 10 compares numerical values of the generalized stress intensity factor using the $\mathrm{AT}_{1}$ model with experimental measurements, and the actual numerical values are included in Table A.8 and A.9.
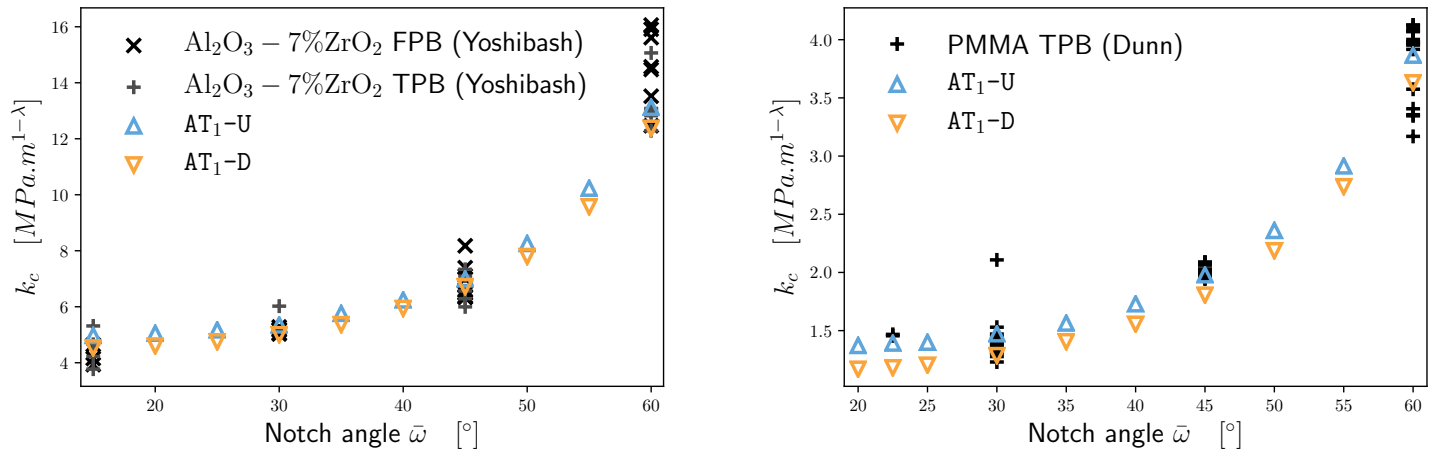

Figure 10: Critical generalized stress intensity factor $k_{c}$ vs notch angle for $\mathrm{Al}_{2} \mathrm{O}_{3}-7 \% \mathrm{ZrO}_{2}$ (left) and PMMA (right). The black markers represents all experimental results. The numerical results are obtained through the Pac-Man test using the $\mathrm{AT}_{1}$ model. See Tables A.8-A.9 in the Appendix for the raw data.

For the Aluminium oxyde-Zirconia ceramic, we observe that the absolute error between measurement and numerical prediction is typically well within the standard deviation of the experimental measurement. As expected, damaged notch boundary conditions lead to better approximation of $k_{c}$ for small angles, and undamaged notches are better for larger values of $\bar{\omega}$.

For the three point bending experiments in PMMA of [38] later reported in [92], the experimental results suggest that the relative depth $a / h$ of the notch has a significant impact on $k_{c}$. We then performed full-domain numerical simulation using the geometry and loading from the literature, and compared the critical force upon which a crack nucleates in experiments and simulations. All computations were performed using the $\mathrm{AT}_{1}$ model in plane strain with undamaged notch boundary conditions. Figure 11 compares the experimental and simulated value of the critical load at failure, listed in Table A.10 and A.11.

These simulations show that a robust quantitative prediction of the failure load in geometries involving a broad range of stress singularity power can be achieved numerically with the $\mathrm{AT}_{1}$ model, provided that the internal length be computed using (6), which involves only material properties. In other words, our approach is capable of predicting crack nucleation near a weak stress singularity using only elastic properties, fracture toughness $G_{c}$, the tensile strength $\sigma_{c}$, and the local energy minimization principle (3).

In light of Figure 11, we suggest that both toughness and tensile strength (or equivalently toughness and internal length) can be measured by matching full domain or Pac-Man computations and experiments involving weak elastic singularity of various power (TPB, FPB, SENT, DENT with varying 

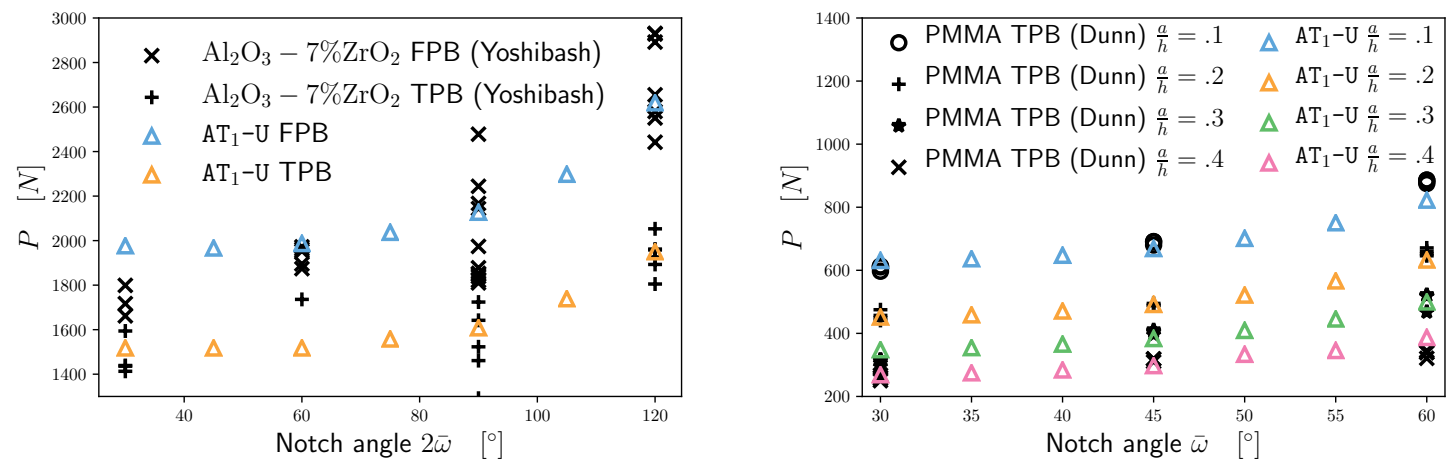

Figure 11: Critical load in the three- and four-point bending experiments of a $\mathrm{Al}_{2} \mathrm{O}_{3}-7 \% \mathrm{ZrO}_{2}$ sample (left) and four-point bending of a PMMA sample (right) from [92] compared with numerical simulations using the $\mathrm{AT}_{1}$ model and undamaged notch boundary conditions. Due to significant variations in measurements in the first set of experiments, each data point reported in [92] is plotted. For the PMMA experiments, average values are plotted and error bars show the range of measured values. See Table A.10 and A.11 in the Appendix for raw data.

notch depth or angle) instead of measuring $\sigma_{c}$ directly. We expect that this approach be much less sensitive to imperfections that the direct measurement of tensile strength, which is virtually impossible. Furthermore, since our criterion is not based on crack tip asymptotics, using full domain computations do not require that the experiments be specially designed to isolated the notch tip singularity from structural scale deformations.

\subsection{Initiation near a stress concentration: the U-notch}

Crack nucleation in a U-shaped notch is another classical problem that has attracted a wealth of experimental and theoretical work. Consider a U-shaped notch of width $\rho$ length $a \gg \rho$ subject to a mode-I local loading (see Figure 12 for a description of notch geometry in the context of a double edge notch tension sample). Assuming "smooth" loadings and applied boundary displacements, elliptic regularity mandates that the stress field be non-singular near the notch tip, provided that $\rho>0$. Within the realm of Griffith fracture, this of course makes crack nucleation impossible. As it is the case for the V-notch, introducing a nucleation principle based on a critical stress is also not satisfying as it will lead to a nucleation load going to 0 as $\rho \rightarrow 0$, instead of converging to that of an infinitely thin crack given by Griffith's criterion. There is a significant body of literature on "notch mechanics", seeking to address this problem introducing stress based criteria, generalized stress intensity factors, or intrinsic material length and cohesive zones. A survey of such models, compared with experiments on a wide range of brittle materials is given [48].

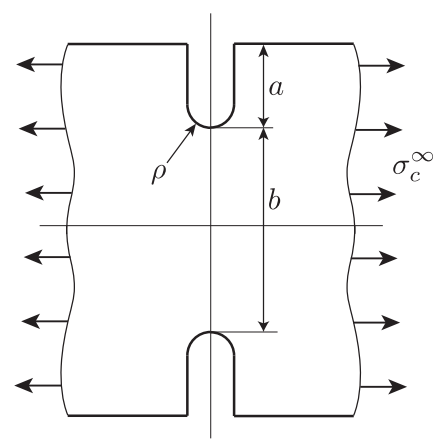

Figure 12: DENT geometry

In what follows, we study crack nucleation near stress concentrations in the $\mathrm{AT}_{1}$ and $\mathrm{AT}_{2}$ models and compare with the experiments gathered in [48]. The core of their analysis consist in defining a 
generalized stress intensity factor

$$
K_{U}=K_{t} \sigma_{c}^{\infty} \sqrt{\frac{\pi \rho}{4}}
$$

where $K_{t}$, the notch stress concentration factor, is a parameter depending on the local $(a$ and $\rho)$, as well as global sample geometry and loading. Through a dimensional analysis, they studied the dependence of the critical generalized stress intensity factor at the onset of fracture and the notch radius. We performed series of numerical simulations of double edge tension (DENT) experiments on a sample of length $h=40$ for multiple values of the notch depth $a$, radius $\rho$, and spacing $b$ given in Table 3 , for which the values of $K_{t}$ were computed in [60]. In each case, we leveraged the symmetries of the problem by performing computations with the $\mathrm{AT}_{1}$ and $\mathrm{AT}_{2}$ models on a quarter of the domain for a number of values of the internal length $\ell$ corresponding to $\rho / \ell_{c h}$ between 0.05 and 20 . In all cases, undamaged notch boundary conditions were used.

\begin{tabular}{ccccc}
\hline Notch shape & Notch depth $a$ & Notch tip radius $\rho$ & Ligament width $b$ & $K_{t}$ \\
\hline $\mathrm{U}$ & 10 & 2.5 & 20 & 5.33 \\
$\mathrm{U}$ & 10 & 1.25 & 20 & 7.26 \\
$\mathrm{U}$ & 10 & 0.5 & 20 & 11.12 \\
\hline
\end{tabular}

Table 3: DENT sample geometry.
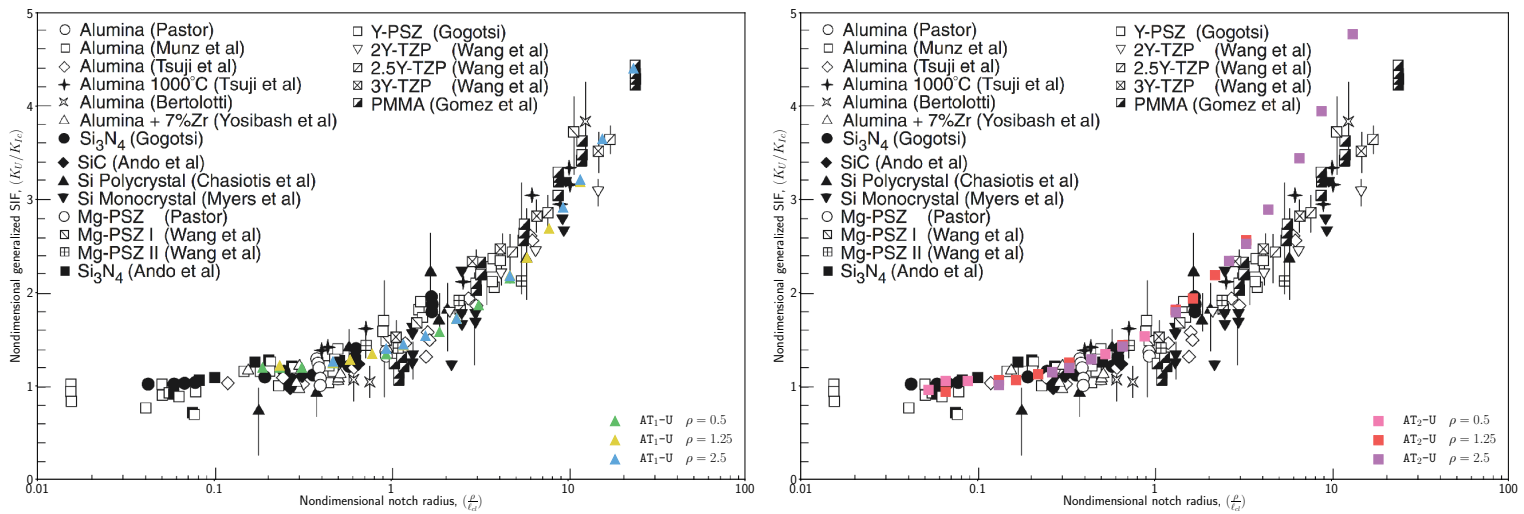

Figure 13: Crack nucleation at U-notches. Comparison between experimental data of [48] and numerical simulations using the $\mathrm{AT}_{1}$ (left) and $\mathrm{AT}_{2}$ (right) models.

In Figure 13, we overlay the outcome of our simulations over the experimental results gathered in [48]. As for the $\mathrm{V}$-notch, we observe that the $\mathrm{AT}_{2}$ model performs poorly for weak stress concentrations (large values of $\rho / \ell_{c h}$ ), as the lack of an elastic phase leads to the creation of large partially damaged areas. For sharp notches $(\rho \simeq 0)$, our simulations concur with the experiments in predicting crack nucleation when $K_{U}=K_{I c}$. As see earlier, the $\mathrm{AT}_{1}$ slightly overestimates the critical load in this regime when undamaged notch boundary conditions are used. In light of 13, we claim that numerical simulations based on the variational phase-field model $\mathrm{AT}_{1}$ provides a simple way to predict crack nucleation that does not require the computation of a notch stress concentration factors $K_{t}$ or the introduction of an ad-hoc criterion.

\section{Size effects in variational phase-field models}

Variational phase-field models are characterised by the intrinsic length $\ell$, or $\ell_{c h}$. In this section, we show that this length-scale introduces physically pertinent scale effects, corroborating its interpretation as a material length. To this end, we study the nucleation of a crack in the uniaxial traction of a plate $(-W, W) \times(-L, L)$ with a centered elliptical hole with semi-axes $a$ and $\rho a(0 \leq \rho \leq 1)$ along the $x$ - 
and $y$-axes respectively, see Figure 14. In Section 4.1, we study the effect of the size and shape of the cavity, assumed to be small with respect to the dimension of the plate $(a \ll W, L)$. In Section 4.2 , we investigate material and structural size effects for a plate of finite width in the limit case of a perfect crack $(\rho=0)$.

\subsection{Effect of an elliptical cavity: size and shape effects}
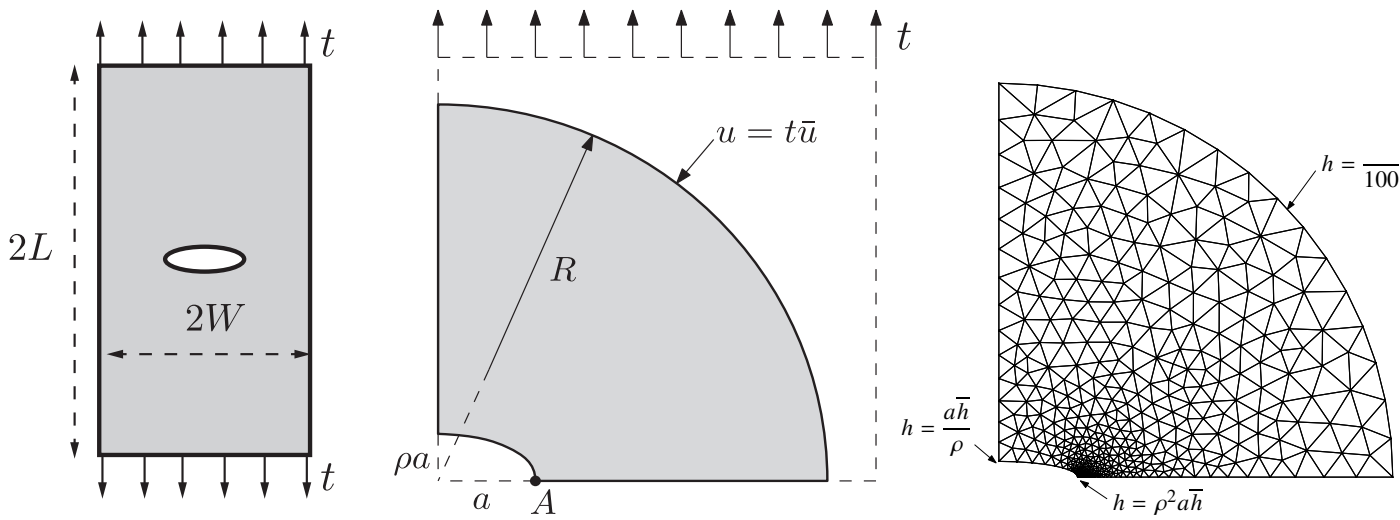

Figure 14: Crack nucleation in an infinite domain containing an elliptical hole. (left) domain geometry (centre) computational domain (right) typical mesh.

For a small hole $(a \ll W, L)$, up to a change of scale, the problem can be fully characterized by two dimensionless parameters: $a / \ell$, and $\rho$. For a linear elastic and isotropic material ocuppying an infinite domain, a close form expression of the stress field as a function of the hole size and aspect ratio is given in [54]. The stress is maximum at the points $A=(a, 0)$ and $A^{\prime}=(-a, 0)$, where the radial stress is zero and the hoop stress is given by:

$$
\sigma_{\max }=t\left(1+\frac{2}{\rho}\right)
$$

$t$ denoting the applied tensile stress along the upper and lower edges of the domain, i.e. the applied macroscopic stress at infinity. We denote by $\bar{u}$ the corresponding displacement field for $t=1$, which is given in [44].

As for the case of a perfect bar, (14) exposes a fundamental issue: if $\rho>0$, the stress remains finite, so that Griffith-based theories will only predict crack nucleation if $\rho=0$. In that case the limit load given by the Griffith's criterion for crack nucleation is

$$
t=\sigma_{G}:=\sqrt{\frac{G_{c} E^{\prime}}{a \pi}}
$$

However, as $\rho \rightarrow 0$, the stress becomes singular so that the critical tensile stress $\sigma_{c}$ is exceeded for an infinitesimally small macroscopic stress $t$.

Following the findings of the previous sections, we focus our attention on the $\mathrm{AT}_{1}$ model only, and present numerical simulations assuming a Poisson ratio $\nu=0.3$ and plane-stress conditions. We perform our simulations in domain of finite size, here a disk of radius $R$ centered around the defect. Along the outer perimeter of the domain, we apply a boundary displacement $u=t \bar{u}$, where $\bar{u}$ is as in [54], and we use the macroscopic stress $t$ a loading parameter. Assuming a symmetric solution, we perform our computations on a quarter domain. For the circular case $\rho=1$, we use a reference mesh size $\bar{h}=\ell_{\min } / 10$, where $\ell_{\min }$ is the smallest value of the internal length of the set of simulations. For 
$\rho<1$, we selectively refine the element size near the expected nucleation site (see Figure 14-right). In order to minimize the effect of the finite size of the domain, we set $R=100 a$.

We performed numerical simulations varying the aspect ratio $a / \ell$ from 0.1 to 50 and the ellipticity $\rho$ from 0.1 to 1.0. In each case, we started from an undamaged state an monotonically increased the loading. In all numerical simulations, we observe two critical loading $t_{e}$ and $t_{c}$, the elastic limit and structural strength, respectively. For $0 \leq t<t_{e}$ the solution is purely elastic, i.e. the damage field $\alpha$ remains identically 0 (see Figure 15-left). For $t_{e} \leq t<t_{c}$, partial distributed damage is observed. The damage field takes its maximum value $\alpha_{\max }<1$ near point $A$ (see Figure 15-center). At $t=t_{c}$, a fully developed crack nucleates, then propagates for $t>t_{c}$ (see Figure 15-right). As for the Pac-Man problem, we identify the crack nucleation with a jump in surface energy, and focus on loading at the onset of damage.
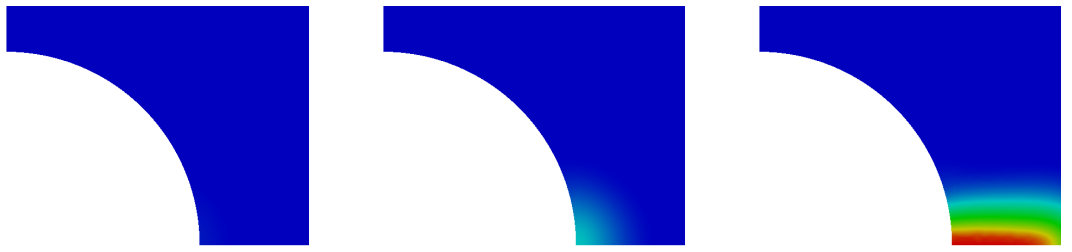

Figure 15: Damage field at the boundary of the hole in the elastic phase $0<t<t_{e}$ (left), the phase with partial damage $t_{e}<t<t_{c}$ (center), and after the nucleation of a crack $t>t_{c}$ (right). Blue: $\alpha=0$, red: $\alpha=1$. The simulation is for $\rho=1.0$ and $a / \ell=5$.

From the one-dimensional problem of Section 2.2 and $[77,80]$, we expect damage nucleation to take place when the maximum stress $\sigma_{\max }$ reaches the nominal material strength $\sigma_{c}=\sqrt{3 G_{c} E^{\prime} / 8 \ell}$ (see Table 1), i.e. for a critical load

$$
t_{e}=\frac{\rho}{2+\rho} \sigma_{c}=\frac{\rho}{2+\rho} \sqrt{\frac{3 G_{c} E^{\prime}}{8 \ell}} .
$$

Figure 16-left confirms this expectation by comparing the ratio $t_{e} / \sigma_{c}$ to its expected value $\rho /(2+\rho)$ for $\rho$ ranging from 0.1 to 1 . Figure 16-right highlights the absence of size effect on the damage nucleation load, by comparing $t_{e} / \sigma_{c}$ for multiple values of $a / \ell$ while keeping $\rho$ fixed at 0.1 and 1 .

Figure 17 focuses on the crack nucleation load $t_{c}$, showing its dependence on the defect shape (left) and size (right). Figure 17-right shows the case of circular hole $(\rho=1)$ and an elongated ellipse, which can be identified to a crack $(\rho=0.1)$. It clearly highlights a scale effect including three regimes:

i. For "small" holes $(a \ll \ell)$, crack nucleation takes place when $t=\sigma_{c}$, as in the uniaxial traction of a perfect bar without the hole: the hole has virtually no effect on crack nucleation. In this regime the strength of a structure is completely determined by that of the constitutive material. Defects of this size do not reduce the structural strength and can be ignored at the macroscopic level.

ii. Holes with length of the order of the internal length $(a=\mathcal{O}(\ell))$, have a strong impact on the structural strength. In this regime the structural strength can be approximated by

$$
\log \left(t_{c} / \sigma_{c}\right)=D \log (a / \ell)+c,
$$

where $D$ is an dimensionless coefficient depending on the defect shape. For a circular hole $\rho=1$, we have $D \approx-1 / 3$.

iii. When $a \gg \ell$, the structural failure is completely determined by the stress distribution surrounding the defect. We observe that for weak stress singularities $(\rho \equiv 1)$, nucleation takes place when the maximum stress reaches the elastic limit $\sigma_{e}$, whereas the behavior as $\rho \equiv 0$ is consistent with Griffith criterion, i.e. the nucleation load scales as $1 / \sqrt{a}$.

Figure 17-right shows that the shape of the cavity has a significative influence on the critical load only in the latter regime, $a \gg \ell$. Indeed, for $a / \ell$ of the order of the unity or smaller, the critical loads $t_{c}$ for circular and highly elongated cavities are almost indistinguishable. This small sensitivity 

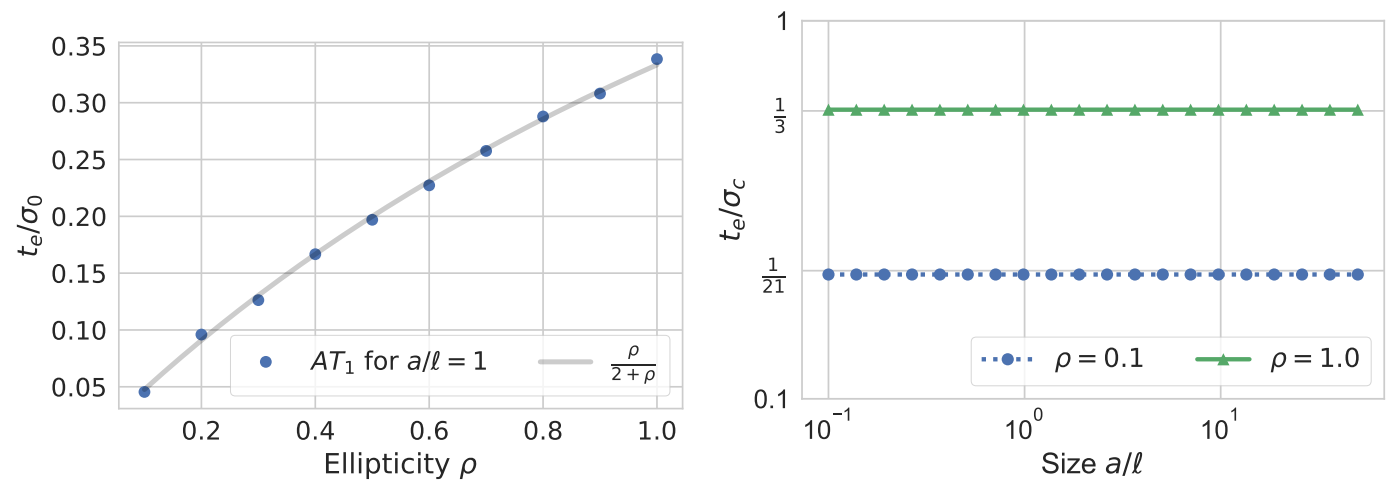

Figure 16: Normalized applied macroscopic stress $t_{e} / \sigma_{c}$ at damage initiation as a function of the aspect ratio $\rho$ for $a / \ell=1$ (left) and of the relative defect sizes $a / \ell$ for $\rho=1$ and $\rho=0.1$ (right).
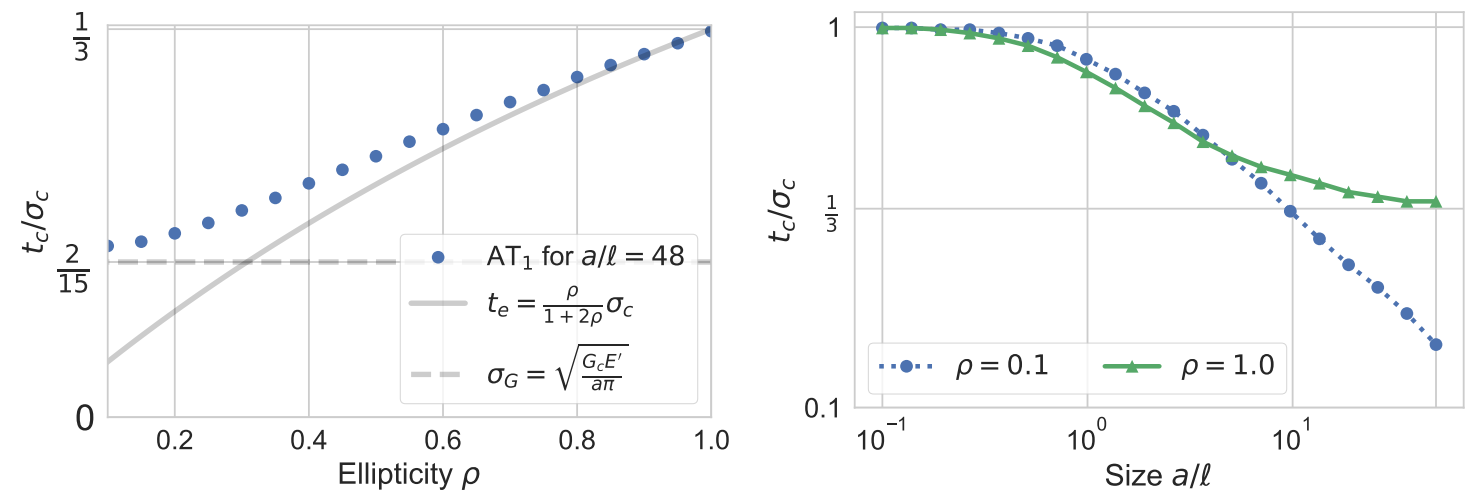

Figure 17: Normalized applied macroscopic stress $t_{c} / \sigma_{e}$ at crack nucleation for an elliptic cavity in an infinite plate. Left: shape effect for cavities of size much larger than the internal length $(a / \ell=48)$; the solid line is the macroscopic stress at the damage initiation $t_{e}$ (see also Figure 16) and dots are the numerical results for the $\mathrm{AT}_{1}$ model. Right: size effect for circular $(\rho=1.0)$ and highly elongated $(\rho=0.1)$ cavities.

of the critical load on the shape is the result of the stress-smoothing effect of the damage field, which is characterised by a cut-off length of the order of $\ell$. Figure 17-left shows the critical stress $t_{c}$ at nucleation when varying the aspect ratio $\rho$ for $a / \ell=48$, for which $\sigma_{G} / \sigma_{c}=2 / 15$. As expected, the critical stress varies smoothly from the value $\sigma_{G}$ (15) predicted by the Griffith theory for a highly elongated cavity identified to a perfect crack, to $t_{e}(16)$ for circular cracks, where the crack nucleates as soon as the maximum stress $\sigma_{\max }$ attains the elastic limit.

This series of experiments is consistent with the results of Section 3.2 showing that variational phase-field models are capable of simultaneously accounting for critical elastic energy release rate and critical stress. Furthermore, they illustrate how the internal length $\ell$ can be linked to critical defect size as the nucleation load for a vanishing defect of size less than $\ell$ approaches that of a flawless structure.

\subsection{Competition between material and structural size effects}

We can finally conclude the study of size effects in variational phase-field models by focusing on the competition between material and structural size effects. For that matter, we study the limit case $\rho=0$ of a perfect crack of finite length $2 a$ in a plate of finite width $2 W$, see Figure 18-left. Under the hypotheses of LEFM, the critical load upon which the crack propagates is

$$
\sigma_{G}\left(a / \ell_{c h}, a / W\right)=\sqrt{\frac{G_{c} E^{\prime} \cos \left(\frac{a \pi}{2 W}\right)}{a \pi}}=\sigma_{c} \sqrt{\frac{1}{\pi} \frac{\ell_{c h}}{a} \cos \left(\frac{a \pi}{2 W}\right)} .
$$



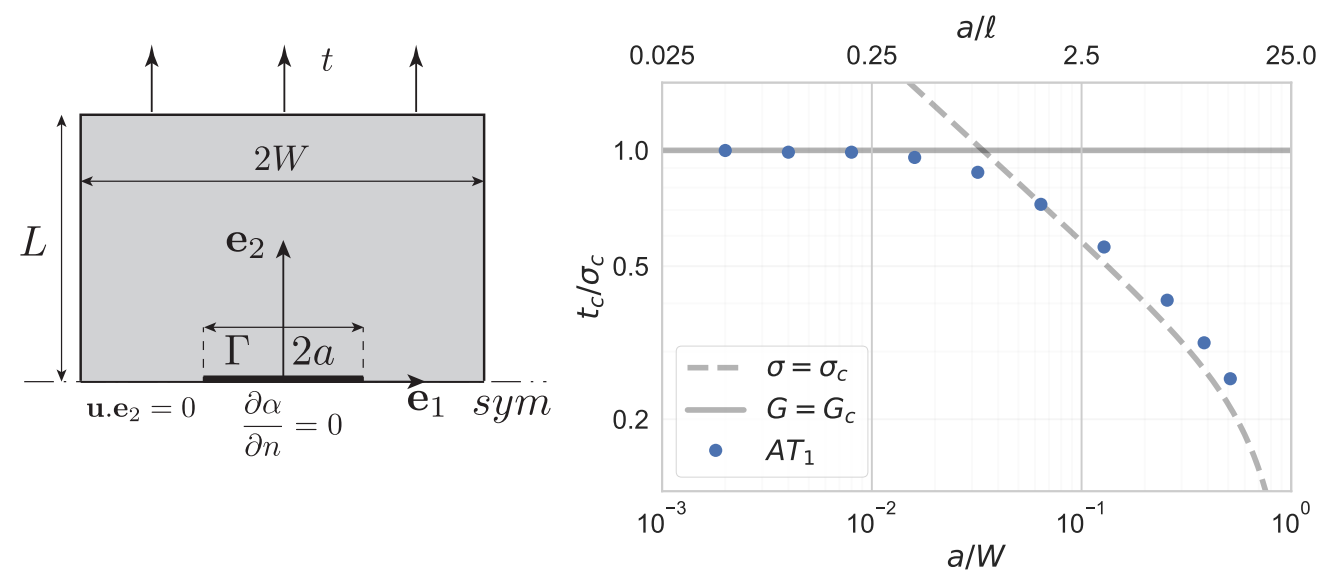

Figure 18: Initiation of a crack of length $2 a$ in a plate of finite width $2 W$. The numerical results (dots) are obtained with the $\mathrm{AT}_{1}$ model for $\ell=W / 25$. The strength criterion and the Griffith's criterion (18).

which reduces to (15) for large plate $(W / a \rightarrow \infty)$. As before, we note that $\sigma_{G} / \sigma_{c} \rightarrow \infty$ as $a / \ell_{c h} \rightarrow 0$, so that for any given load, the material's tensile strength is exceeded for short enough initial crack.

We performed series of numerical simulations using the $\mathrm{AT}_{1}$ a model on the a quarter of the domain with $W=1, L=4, \nu=0.3, \ell=W / 25, h=\ell / 20$, and the initial crack's half-length $a$ ranging from from $0.025 \ell$ to $12.5 \ell$ (i.e. $0.001 \mathrm{~W}$ to $0.5 \mathrm{~W}$ ). The pre-existing crack was modelled as a geometric feature and undamaged crack lip boundary conditions were prescribed. The loading was applied by imposing a uniform normal stress of amplitude $t$ to its upper and lower edge.

Figure 18, displays the normalized macroscopic structural strength of the sample, $t_{c} / \sigma_{c}$, where $\sigma_{c}$ is given by (6), and $t_{c}$ is the applied load upon which the crack grows, identified as before. The results are in good agreement with classical theories linking size-effect on the strength of the material [11]. When $a \gg \ell$, i.e. when the defect is large compared to the material's length, crack initiation is governed by Griffith's criterion (18). As noted earlier, the choice of undamaged notch boundary conditions on the damage fields leads to slightly overestimating the nucleation load. Our numerical simulations reproduce the structural size effect predicted by LEFM when the crack length is comparable to the plate width $W$.

When $a \ll \ell$ however, we observe that the macroscopic structural strength is very close to the material's tensile strength. Again, below the material's internal length, defects have virtually no impact on the structural response. LEFM and Griffith-based models cannot account for this material size-effect. These effects are introduced in variational phase-field model by the additional material parameter $\ell$.

In the intermediate regime $a=\mathcal{O}(\ell)$, we observe a smooth transition between strength and toughness criteria, where the tensile strength is never exceeded.

When $a \gg \ell$, our numerical simulations are consistent with predictions from Linear Elastic Fracture Mechanics shown as a dashed line in Figure 18, whereas when $a \ll \ell$, the structural effect of the small crack disappear, and nucleation takes place at or near the material's tensile strength, i.e. $t_{c} / \sigma_{c} \simeq 1$.

\section{Conclusion}

In contrast with most of the literature on phase-field models of fracture focusing validation and verification in the context of propagation "macroscopic" cracks [69, 81], we have studied crack nucleation.

In this article, we have studied crack nucleation and initiation in multiple geometries. We confirmed observations reported elsewhere in the literature that although they are mathematically equivalent in the limit of $\ell \rightarrow 0$, damaged notch boundary conditions lead to a more accurate computation near strong stress singularities whereas away from singularities, undamaged notch boundary conditions are 
to be used. Our numerical simulations also highlight the superiority of phase-fiel models such as $\mathrm{AT}_{1}$ which exhibit an elastic phase in the one-dimensional tension problem over those who don't (such as $\mathrm{AT}_{2}$ ), when nucleation away from strong singularity is involved. Our numerical simulations suggest that it is not possible to accurately account for crack nucleation near "weak" singularities using the $\mathrm{AT}_{2}$ model. We infer that a strictly positive elastic limit $\sigma_{e}$ is a required feature of a phase-field model that properly account for crack nucleation.

We have shown that as suggested by the one-dimensional tension problem, the regularization parameter $\ell$ must be understood (up to a model-dependent multiplicative constant) as the material's characteristic or internal length $\ell_{c h}=G_{c} E / \sigma_{c}^{2}$, and linked to the material strength $\sigma_{c}$. With this adjustment, we show that variational phase-field models are capable of quantitative prediction of crack nucleation in a wide range of geometries including three- and four-point bending with various type of notches, single and double edge notch tests, and a range of brittle materials, including steel and duraluminium at low temperatures, PVC foams, PMMA, and several ceramics.

We recognize that measuring a material's tensile strength is difficult and sensitive to the presence of defect, so that formulas (6) may not be a practical way of computing a material's internal length. Instead, we propose to perform series of experiments such as three point bending with varying notch depth, radius or angle, as we have demonstrated in Figure 11 that with a properly adjusted internal length, variational phase-field models are capable of predicting the nucleation load for any notch depth or aperture. Furthermore, since variational phase-field models do not rely on any crack-tip asymptotic, this identification can be made even in situation where generalized stress or notch intensity factors are not known, or are affected by the sample's structural geometry.

We have also shown that variational phase-field models properly account for size effects that cannot be recovered from Griffith-based theories. By introducing the material's internal length, they can account for the vanishing effect of small defects on the structural response of a material, or reconcile the existence of a critical material strength with the existence of stress singularity. Most importantly, they do not require introducing series of ad-hoc criteria based on local geometry and loading. On the contrary, we see that in most situation, criteria derived from the asymptotic analysis of a microgeometry can be recovered a posteriori. Furthermore, variational phase-field models are capable of quantitative prediction of crack path after nucleation. Again, they do so without resolving to introducing additional ad-hoc criteria, but only relying on a general energy minimisation principle.

In short, we have demonstrated that variational phase-field models address some of the most vexing issues associated with brittle fracture: scale effects, nucleation, existence of a critical stress, and path prediction.

Of course, there are still remaining issues that need to be addressed. Whereas the models are derived from irreversibility, stability and energy balance, our numerical simulations do not enforce energy balance as indicated by a drop of the total energy upon crack nucleation without string singularities. Note that to this day, devising an evolution principle combining the strength of (3) while ensuring energy balance is still an open problem. Perhaps extensions into phase field models dynamic fracture will address this issue.

Fracture in compression remains an issue in variational phase-field models. Although several approaches have been proposed that typically consist in splitting the strain energy into a damage inducing and non damage inducing terms, neither of the proposed splits are fully satisfying (see [64] for instance). In particular, it is not clear of either of this models is capable of simultaneously accounting for nucleation under compression and self-contact.

Finally, even though a significant amount of work has already been invested in extending the scope of phase-field models of fracture beyond perfectly brittle materials, to our knowledge, none of the proposed extension has demonstrated its predictive power yet.

\section{Acknowledgments}

This material is based upon work supported by the National Science Foundation under Grant No. DMS-1312739 and DMS-1535076. This work used the Extreme Science and Engineering Discovery Environment (XSEDE), which is supported by National Science Foundation grant number ACI-1548562. 
Portions of this research were conducted with high performance computing resources provided by Louisiana State University (http://www.hpc.Isu.edu) and by the institute for computing and data sciences (ISCD) of University Pierre et Marie Curie (http://iscd.upmc.fr). CM acknowledges the support of the project ANR-11-LABX-0037 (Agence Nationale de la Recherche) and of Sorbonnes Universités project FRZX026-SU-16-R-EMR-02-ANIS.

\section{Appendix A. Tables of experimental an numerical data for V-notch experiments}

\begin{tabular}{llllllll}
\hline & \multicolumn{3}{c}{ Experiments } & \multicolumn{2}{c}{ Undamaged notch } & \multicolumn{2}{c}{ Damaged notch } \\
$2 \bar{\omega}$ & Type & $k_{c}^{(\text {exp })}$ & stdev & $k_{c}^{(\text {num })}$ & rel. error & $k_{c}^{(\text {num })}$ & rel. error \\
\hline $0^{\circ}$ & TPB & 51.77 & 3.06 & 67.09 & $22.84 \%$ & 54.69 & $5.35 \%$ \\
$30^{\circ}$ & SENT & 60.97 & 1.97 & 66.91 & $8.88 \%$ & 56.99 & $6.98 \%$ \\
$60^{\circ}$ & SENT & 65.81 & 1.52 & 69.55 & $5.39 \%$ & 62.95 & $4.53 \%$ \\
$90^{\circ}$ & TPB & 88.62 & 3.58 & 85.16 & $4.06 \%$ & 78.15 & $13.40 \%$ \\
$120^{\circ}$ & SENT & 142.74 & 2.25 & 130.81 & $9.12 \%$ & 121.68 & $17.30 \%$ \\
$140^{\circ}$ & SENT & 243.73 & 31.86 & 211.06 & $15.48 \%$ & 191.91 & $27.00 \%$ \\
\hline
\end{tabular}

Table A.4: Generalized critical stress intensity factors as a function of the notch aperture in soft annealed tool steel, (AISI O1 at $-50^{\circ} \mathrm{C}$ ). Experimental measurements from [88] using SENT and TPB compared with Pac-Man simulations with the $\mathrm{AT}_{1}$ model.

\begin{tabular}{|c|c|c|c|c|c|c|c|}
\hline \multirow[b]{2}{*}{$2 \bar{\omega}$} & \multirow[b]{2}{*}{ Mat } & \multicolumn{2}{|c|}{ Experiments } & \multicolumn{2}{|c|}{ Undamaged notch } & \multicolumn{2}{|c|}{ Damaged notch } \\
\hline & & $k_{c}^{(e x p)}$ & stdev & $k_{c}^{(\text {num })}$ & rel. error & $k_{c}^{(n u m)}$ & rel. error \\
\hline \multirow[t]{4}{*}{$0^{\circ}$} & $\mathrm{H} 80$ & 0.14 & 0.01 & 0.18 & $22.91 \%$ & 0.15 & $5.81 \%$ \\
\hline & H100 & 0.26 & 0.02 & 0.34 & $24.62 \%$ & 0.28 & $7.61 \%$ \\
\hline & H130 & 0.34 & 0.01 & 0.44 & $29.34 \%$ & 0.36 & $5.09 \%$ \\
\hline & H200 & 0.57 & 0.02 & 0.74 & $47.60 \%$ & 0.61 & $6.53 \%$ \\
\hline \multirow[t]{4}{*}{$90^{\circ}$} & H80 & 0.20 & 0.02 & 0.22 & $12.65 \%$ & 0.21 & $4.73 \%$ \\
\hline & H100 & 0.36 & 0.02 & 0.41 & $12.29 \%$ & 0.38 & $4.10 \%$ \\
\hline & H130 & 0.49 & 0.05 & 0.54 & $11.33 \%$ & 0.50 & $0.50 \%$ \\
\hline & H200 & 0.81 & 0.08 & 0.91 & $20.54 \%$ & 0.83 & $2.21 \%$ \\
\hline \multirow[t]{4}{*}{$140^{\circ}$} & H80 & 0.53 & 0.06 & 0.53 & $0.37 \%$ & 0.48 & $9.26 \%$ \\
\hline & H100 & 0.89 & 0.04 & 0.92 & $3.43 \%$ & 0.84 & $5.91 \%$ \\
\hline & H130 & 1.22 & 0.10 & 1.25 & $2.95 \%$ & 1.13 & $7.48 \%$ \\
\hline & $\mathrm{H} 200$ & 2.02 & 0.14 & 2.07 & $4.92 \%$ & 1.89 & $6.80 \%$ \\
\hline \multirow[t]{4}{*}{$155^{\circ}$} & $\mathrm{H} 80$ & 0.86 & 0.07 & 0.83 & $3.63 \%$ & 0.75 & $14.36 \%$ \\
\hline & H100 & 1.42 & 0.08 & 1.42 & $0.14 \%$ & 1.29 & $10.63 \%$ \\
\hline & H130 & 1.90 & 0.10 & 1.95 & $2.82 \%$ & 1.76 & $8.06 \%$ \\
\hline & $\mathrm{H} 200$ & 3.24 & 0.15 & 3.23 & $0.89 \%$ & 2.92 & $11.02 \%$ \\
\hline
\end{tabular}

Table A.5: Generalized critical stress intensity factors as a function of the notch aperture in Divinycell@ PVC foam. Experimental measurements from [50] using four point bending compared with Pac-Man simulations with the $\mathrm{AT}_{1}$ model. 


\begin{tabular}{llllllll}
\hline & \multicolumn{3}{c}{ Experiments } & \multicolumn{2}{c}{ Undamaged notch } & \multicolumn{2}{c}{ Damaged notch } \\
$\bar{\omega}$ & Type & $k_{c}^{(\text {exp })}$ & stdev & $k_{c}^{(\text {num })}$ & rel. error & $k_{c}^{(\text {(num })}$ & rel. error \\
\hline $10^{\circ}$ & DENT & 53.55 & 0.94 & 64.80 & $17.36 \%$ & 56.40 & $5.05 \%$ \\
$20^{\circ}$ & DENT & 57.10 & 0.26 & 65.11 & $12.30 \%$ & 58.52 & $2.43 \%$ \\
$30^{\circ}$ & DENT & 60.50 & 0.60 & 67.17 & $9.94 \%$ & 62.11 & $2.59 \%$ \\
$40^{\circ}$ & DENT & 66.34 & 0.50 & 74.07 & $10.44 \%$ & 69.24 & $4.18 \%$ \\
$50^{\circ}$ & DENT & 80.15 & 0.46 & 86.61 & $7.46 \%$ & 82.25 & $2.55 \%$ \\
$60^{\circ}$ & DENT & 102.00 & 1.17 & 114.20 & $10.69 \%$ & 107.43 & $5.05 \%$ \\
$70^{\circ}$ & DENT & 150.44 & 1.17 & 170.19 & $11.61 \%$ & 158.91 & $5.33 \%$ \\
$80^{\circ}$ & DENT & 291.75 & 1.94 & 305.03 & $4.35 \%$ & 274.74 & $6.19 \%$ \\
$90^{\circ}$ & DENT & 705.27 & 8.53 & 661.19 & $6.67 \%$ & 592.59 & $19.01 \%$ \\
\hline
\end{tabular}

Table A.6: Generalized critical stress intensity factors as a function of the notch aperture in duraluminium. Experimental measurements from [85] using single edge notch tension compared with Pac-Man simulations with the $\mathrm{AT}_{1}$ model.

\begin{tabular}{llllllll}
\hline & & \multicolumn{3}{c}{ Experiments } & \multicolumn{2}{c}{ Undamaged notch } & \multicolumn{2}{c}{ Damaged notch } \\
$\bar{\omega}$ & Type & $k_{c}^{(\text {exp })}$ & stdev & $k_{c}^{(\text {num })}$ & rel. error & $k_{c}^{(\text {num })}$ & rel. error \\
\hline $10^{\circ}$ & DENT & 1.87 & 0.03 & 2.50 & $25.29 \%$ & 2.07 & $10.03 \%$ \\
$20^{\circ}$ & DENT & 1.85 & 0.03 & 2.53 & $26.89 \%$ & 2.13 & $12.97 \%$ \\
$30^{\circ}$ & DENT & 2.17 & 0.03 & 2.65 & $18.17 \%$ & 2.33 & $6.92 \%$ \\
$40^{\circ}$ & DENT & 2.44 & 0.02 & 3.07 & $20.65 \%$ & 2.73 & $10.70 \%$ \\
$50^{\circ}$ & DENT & 3.06 & 0.05 & 3.94 & $22.31 \%$ & 3.54 & $13.63 \%$ \\
$60^{\circ}$ & DENT & 4.35 & 0.18 & 5.95 & $26.97 \%$ & 5.41 & $19.69 \%$ \\
$70^{\circ}$ & DENT & 8.86 & 0.18 & 11.18 & $20.74 \%$ & 10.10 & $12.26 \%$ \\
$80^{\circ}$ & DENT & 28.62 & 0.68 & 27.73 & $3.20 \%$ & 24.55 & $16.56 \%$ \\
$90^{\circ}$ & DENT & 104.85 & 10.82 & 96.99 & $8.11 \%$ & 85.37 & $22.82 \%$ \\
\hline
\end{tabular}

Table A.7: Generalized critical stress intensity factors as a function of the notch aperture in PMMA. Experimental measurements from [85] using single edge notch tension compared with Pac-Man simulations with the AT 1 model.

\begin{tabular}{llllllll}
\hline & & \multicolumn{2}{c}{ Experiments } & \multicolumn{2}{c}{ Undamaged notch } & \multicolumn{2}{c}{ Damaged notch } \\
$2 \bar{\omega}$ & type & $k_{c}^{(\text {(exp })}$ & stdev & $k_{c}^{(\text {num })}$ & rel. error & $k_{c}^{(\text {num })}$ & rel. error \\
\hline $30^{\circ}$ & TPB & 4.49 & 0.57 & 4.97 & $9.6 \%$ & 4.53 & $0.9 \%$ \\
& FPB & 4.24 & 0.30 & 4.97 & $14.6 \%$ & 4.53 & $6.4 \%$ \\
$60^{\circ}$ & TPB & 6.02 & n/a & 5.35 & $12.6 \%$ & 5.00 & $20.3 \%$ \\
& FPB & 5.14 & 0.09 & 5.35 & $3.8 \%$ & 5.00 & $2.8 \%$ \\
$90^{\circ}$ & TPB & 6.66 & 0.50 & 6.99 & $4.8 \%$ & 6.72 & $1.0 \%$ \\
& FPB & 6.81 & 0.54 & 6.99 & $2.6 \%$ & 6.72 & $1.3 \%$ \\
$120^{\circ}$ & TPB & 13.21 & 0.87 & 13.12 & $0.7 \%$ & 12.38 & $6.8 \%$ \\
& FPB & 14.66 & 1.23 & 13.12 & $11.7 \%$ & 12.38 & $18.4 \%$ \\
\hline
\end{tabular}

Table A.8: Generalized critical stress intensity factors as a function of the notch aperture in Aluminium oxyde ceramics. Experimental measurements from [92] using three and four point bending compared with Pac-Man simulations. 


\begin{tabular}{llllllll}
\hline & \multicolumn{6}{c}{ Experiments } & \multicolumn{2}{c}{ Undamaged notch } & \multicolumn{2}{c}{ Damaged notch } \\
$2 \bar{\omega}$ & $a / h$ & $k_{c}^{(\text {exp })}$ & stdev & $k_{c}^{(\text {(num })}$ & rel. error & $k_{c}^{(\text {num })}$ & rel. error \\
\hline $60^{\circ}$ & 0.1 & 1.41 & 0.02 & 1.47 & $4.5 \%$ & 1.29 & $9.3 \%$ \\
& 0.2 & 1.47 & 0.04 & 1.47 & $0.4 \%$ & 1.29 & $14.0 \%$ \\
& 0.3 & 1.28 & 0.03 & 1.47 & $13.0 \%$ & 1.29 & $0.4 \%$ \\
& 0.4 & 1.39 & 0.04 & 1.47 & $5.8 \%$ & 1.29 & $7.8 \%$ \\
$90^{\circ}$ & 0.1 & 2.04 & 0.02 & 1.98 & $3.0 \%$ & 1.81 & $12.9 \%$ \\
& 0.2 & 1.98 & 0.01 & 1.98 & $0.0 \%$ & 1.81 & $9.6 \%$ \\
& 0.3 & 2.08 & 0.03 & 1.98 & $5.1 \%$ & 1.81 & $15.2 \%$ \\
$120^{\circ}$ & 0.4 & 2.10 & 0.03 & 1.98 & $5.9 \%$ & 1.81 & $16.1 \%$ \\
& 0.1 & 4.15 & 0.02 & 3.87 & $7.3 \%$ & 3.63 & $14.3 \%$ \\
& 0.2 & 4.03 & 0.06 & 3.87 & $4.2 \%$ & 3.63 & $11.0 \%$ \\
& 0.3 & 3.92 & 0.18 & 3.87 & $1.4 \%$ & 3.63 & $8.0 \%$ \\
& 0.4 & 3.36 & 0.09 & 3.87 & $13.0 \%$ & 3.63 & $7.4 \%$ \\
\hline
\end{tabular}

Table A.9: Generalized critical stress intensity factors as a function of the notch aperture in PMMA. Experimental measurements from [38] using three and four point bending compared with Pac-Man simulations. The value $a / h$ refers to the ratio depth of the notch over sample thickness. See Figure 9 for geometry and loading.

\begin{tabular}{llllll}
\hline $2 \bar{\omega}$ & type & $P_{c}^{(\text {exp })}[\mathrm{N}]$ & stdev & $P_{c}^{(\text {num })}[\mathrm{N}]$ & rel. error \\
\hline $30^{\circ}$ & TPB & 1470.50 & 72.01 & 1517.59 & $3.1 \%$ \\
& FPB & 1726.00 & 56.29 & 1976.59 & $12.7 \%$ \\
$60^{\circ}$ & TPB & 1736.00 & 0.00 & 1517.59 & $14.4 \%$ \\
& FPB & 1909.17 & 60.88 & 1986.62 & $3.9 \%$ \\
$90^{\circ}$ & TPB & 1528.40 & 149.41 & 1608.04 & $5.0 \%$ \\
& FPB & 2024.40 & 212.03 & 2127.09 & $4.8 \%$ \\
$120^{\circ}$ & TPB & 1933.00 & 75.15 & 1949.75 & $0.9 \%$ \\
& FPB & 2711.29 & 187.66 & 2618.73 & $3.5 \%$ \\
\hline
\end{tabular}

Table A.10: Critical load reported in [92] using three- and four-point bending experiments of an $\mathrm{Al}_{2} \mathrm{O}_{3}-7 \% \mathrm{ZrO}_{2}$ sample compared with numerical simulations using the $\mathrm{AT}_{1}$ model and undamaged notch boundary conditions. TPB and $\mathrm{FPB}$ refer respectively to three point bending and four point bending. See Figure 9 for geometry and loading.

\begin{tabular}{llllll}
\hline $2 \bar{\omega}$ & $a / h$ & $P_{c}^{(e x p)}[\mathrm{N}]$ & stdev & $P_{c}^{(\text {num })}[\mathrm{N}]$ & rel. error \\
\hline $60^{\circ}$ & 0.1 & 608.50 & 6.69 & 630.81 & $3.5 \%$ \\
& 0.2 & 455.75 & 12.48 & 451.51 & $0.9 \%$ \\
& 0.3 & 309.00 & 8.19 & 347.98 & $11.2 \%$ \\
& 0.4 & 258.75 & 6.61 & 268.69 & $3.7 \%$ \\
$90^{\circ}$ & 0.1 & 687.33 & 5.19 & 668.69 & $2.8 \%$ \\
& 0.2 & 491.00 & 2.94 & 491.41 & $0.1 \%$ \\
& 0.3 & 404.33 & 5.44 & 383.33 & $5.5 \%$ \\
$120^{\circ}$ & 0.4 & 316.00 & 4.24 & 297.48 & $6.2 \%$ \\
& 0.1 & 881.75 & 4.60 & 822.22 & $7.2 \%$ \\
& 0.2 & 657.25 & 9.36 & 632.32 & $3.9 \%$ \\
& 0.3 & 499.60 & 25.41 & 499.50 & $0.0 \%$ \\
& 0.4 & 336.25 & 9.09 & 386.87 & $13.1 \%$ \\
\hline
\end{tabular}

Table A.11: Load at failure reported in [92] using three point bending experiments of a PMMA sample compared to full domain numerical simulations using the $\mathrm{AT}_{1}$ model with undamaged notch boundary conditions. The value $a / h$ refers to the ratio depth of the notch over sample thickness. See Figure 9 for geometry and loading. 
[1] A. Abdollahi and I. Arias. Phase-field modeling of crack propagation in piezoelectric and ferroelectric materials with different electromechanical crack conditions. J. Mech. Phys. Solids, 60(12):2100 $-2126,2012$.

[2] R. Alessi, J.-J. Marigo, and S. Vidoli. Gradient damage models coupled with plasticity and nucleation of cohesive cracks. Arch. Rat. Mech. Anal., 214(2):575-615, 2014.

[3] M. Ambati, T. Gerasimov, and L. De Lorenzis. Phase-field modeling of ductile fracture. Comput. Mech., 55(5):1017-1040, 2015.

[4] M. Ambati, R. Kruse, and L. De Lorenzis. A phase-field model for ductile fracture at finite strains and its experimental verification. Comput. Mech., 57(1):149-167, nov 2015.

[5] L. Ambrosio and V. M. Tortorelli. Approximation of functionals depending on jumps by elliptic functionals via -convergence. Comm. Pure Appl. Math., 43(8):999-1036, 1990.

[6] L. Ambrosio and V. M. Tortorelli. On the approximation of free discontinuity problems. Boll. Un. Mat. Ital. B (7), 6(1):105-123, 1992.

[7] S. Balay, S. Abhyankar, M. F. Adams, J. Brown, P. Brune, K. Buschelman, L. Dalcin, V. Eijkhout, W. D. Gropp, D. Kaushik, M. G. Knepley, L. Curfman McInnes, K. Rupp, B. F. Smith, S. Zampini, H. Zhang, and H. Zhang. PETSc Web page, 2017.

[8] S. Balay, S. Abhyankar, M. F. Adams, J. Brown, P. Brune, K. Buschelman, L. Dalcin, V. Eijkhout, W. D. Gropp, D. Kaushik, Matthew G. Knepley, L. Curfman McInnes, K. Rupp, B. F. Smith, S. Zampini, H. Zhang, and H. Zhang. PETSc users manual. Technical Report ANL-95/11 Revision 3.7, Argonne National Laboratory, 2016.

[9] S. Balay, W.D. Gropp, L. Curfman McInnes, and B.F. Smith. Efficient management of parallelism in object oriented numerical software libraries. In E. Arge, A. M. Bruaset, and H. P. Langtangen, editors, Modern Software Tools in Scientific Computing, pages 163-202. Birkhäuser Press, 1997.

[10] Z. P. Bažant. Scaling of quasibrittle fracture: asymptotic analysis. Int. J. Fracture, 83(1):19-40, 1997.

[11] Z. P. Bažant. Scaling of Structural Strength. Elsevier, 2nd edition, 2005.

[12] G. Bellettini and A. Coscia. Discrete approximation of a free discontinuity problem. Numer. Funct. Anal. Optim., 15(3-4):201-224, 1994.

[13] A. Benallal and J.-J. Marigo. Bifurcation and stability issues in gradient theories with softening. Modelling and Simulation in Materials Science and Engineering, 15(1):S283, 2007.

[14] M. J. Borden, T. J. R. Hughes, C. M Landis, A. Anvari, and I. J. Lee. A phase-field formulation for fracture in ductile materials: Finite deformation balance law derivation, plastic degradation, and stress triaxiality effects. Comp. Meth. Appl. Mech. Engng., 312:130-166, 2016.

[15] M. J. Borden, T. J.R. Hughes, C. M. Landis, and C. V. Verhoosel. A higher-order phase-field model for brittle fracture: Formulation and analysis within the isogeometric analysis framework. Comp. Methods Appl. Mech. Engng., 273(0):100 - 118, 2014.

[16] B. Bourdin. Image segmentation with a finite element method. M2AN Math. Model. Numer. Anal., 33(2):229-244, 1999.

[17] B. Bourdin. Numerical implementation of a variational formulation of quasi-static brittle fracture. Interfaces Free Bound., 9:411-430, 082007.

[18] B. Bourdin and A. Chambolle. Implementation of an adaptive finite-element approximation of the Mumford-Shah functional. Numer. Math., 85(4):609-646, 2000. 
[19] B. Bourdin, C. Chukwudozie, and K. Yoshioka. A variational approach to the numerical simulation of hydraulic fracturing. In Proceedings of the 2012 SPE Annual Technical Conference and Exhibition, volume SPE 159154, 2012.

[20] B. Bourdin, G. A. Francfort, and J.-J. Marigo. Numerical experiments in revisited brittle fracture. J. Mech. Phys. Solids, 48(4):797-826, 2000.

[21] B. Bourdin, G. A. Francfort, and J.-J. Marigo. The variational approach to fracture. J. Elasticity, 91(1-3):5-148, 2008.

[22] B. Bourdin, J.-J. Marigo, C. Maurini, and P. Sicsic. Morphogenesis and propagation of complex cracks induced by thermal shocks. Phys. Rev. Lett., 112(1):014301, 2014.

[23] A. Braides. Approximation of Free-Discontinuity Problems. Number 1694 in Lecture Notes in Mathematics. Springer, 1998.

[24] A. Braides. $\Gamma$-convergence for beginners, volume 22 of Oxford Lecture Series in Mathematics and its Applications. Oxford University Press, Oxford, 2002.

[25] S. Burke, C. Ortner, and E. Süli. An adaptive finite element approximation of a variational model of brittle fracture. SIAM J. Numer. Anal., 48(3):980-1012, 2010.

[26] S. Burke, C. Ortner, and E. Süli. An adaptive finite element approximation of a generalized Ambrosio-Tortorelli functional. Mathematical Models 83 Methods in Applied Sciences, 23(9):1663 - 1697, 2013.

[27] A. Chambolle. An approximation result for special functions with bounded variations. J. Math Pures Appl., 83:929-954, 2004.

[28] A. Chambolle. Addendum to "an approximation result for special functions with bounded deformation" [j. math. pures appl. (9) 83 (7) (2004) 929-954]: the n-dimensional case. J. Math Pures Appl., 84:137-145, 2005.

[29] M. Charlotte, J. Laverne, and J.-J. Marigo. Initiation of cracks with cohesive force models: a variational approach. Euro J. Mech. A/Solids, 25(4):649-669, 2006.

[30] A. Chudnovsky. Slow crack growth, its modeling and crack-layer approach: A review. International Journal of Engineering Science, 83:6-41, 2014.

[31] C. Chukwudozie. Application of the Variational Fracture Model to Hydraulic Fracturing in Poroelastic Media. PhD thesis, Louisiana State University, Craft \& Hawkins Department of Petroleum Engineering, 2016.

[32] S. Conti, M. Focardi, and F. Iurlano. Phase field approximation of cohesive fracture models. Ann. I. H. Poincaré - AN, 33(4):1033-1067, 2016. preprint.

[33] V. Crismale and G. Lazzaroni. Viscous approximation of quasistatic evolutions for a coupled elastoplastic-damage model. Calc. Var. Partial Differential Equations, 55(17):1-54, 2016.

[34] G. Dal Maso. An introduction to Г-convergence. Birkhäuser, Boston, 1993.

[35] R. de Borst, M. Gutiérrez, G. Wells, J. Remmers, and H. Askes. Cohesive-zone models, higherorder continuum theories and reliability methods for computational failure analysis. Int. J. Numer. Meth. Engng., 60:289-315, 2004.

[36] G. Del Piero, G. Lancioni, and R. March. A diffuse cohesive energy approach to fracture and plasticity: the one-dimensional case. J. Mech. Phys. Solids, 8(2-4):109-151, 2013.

[37] P. Destuynder and M. Djaoua. Sur une interprétation mathématique de l'intégrale de Rice en théorie de la rupture fragile. Math. Methods Appl. Sci., 3(1):70-87, 1981. 
[38] M. L Dunn, W. Suwito, and S. Cunningham. Fracture initiation at sharp notches: correlation using critical stress intensities. Int. J. Solids Struct., 34(29):3873-3883, 1997.

[39] M. L. Falk, A. Needleman, and J. R. Rice. A critical evaluation of cohesive zone models of dynamic fracture. J. Phys $I V, 11(\mathrm{PR} 5): 43-50,2001$.

[40] G.A. Francfort and J.-J. Marigo. Revisiting brittle fracture as an energy minimization problem. J. Mech. Phys. Solids., 46(8):1319-1342, 1998.

[41] F. Fraternali. Free discontinuity finite element models in two-dimensions for in-plane crack problems. Theor. Appl. Fract. Mec., 47(3):274-282, 2007.

[42] F. Freddi and F. Iurlano. Numerical insight of a variational smeared approach to cohesive fracture. J. Mech. Phys. Solids, 98:156-171, 2017.

[43] M. Frémond and B. Nedjar. Damage, gradient of damage and principle of virtual power. Int. J. Solids Struct., 33(8):1083-1103, 1996.

[44] X.-L. Gao. A general solution of an infinite elastic plate with an elliptic hole under biaxial loading. Int. J. Pres. Ves. Pip., 67(1):95-104, 1996.

[45] A. Giacomini. Ambrosio-Tortorelli approximation of quasi-static evolution of brittle fractures. Calc. Var. Partial Differential Equations, 22(2):129-172, 2005.

[46] A. Giacomini and M. Ponsiglione. A discontinuous finite element approximation of quasi-static growth of brittle fractures. Numer. Funct. Anal. Optim., 24(7-8):813-850, 2003.

[47] F. J. Gómez and M. Elices. A fracture criterion for sharp v-notched samples. Int J. Fracture, 123:163-175, 2003.

[48] F. J. Gómez, G. V. Guinea, and M. Elices. Failure criteria for linear elastic materials with U-notches. Int. J. Fracture, 141(1):99-113, 2006.

[49] K. Gou, M. Mallikarjuna, K. R. Rajagopal, and J. R. Walton. Modeling fracture in the context of a strain-limiting theory of elasticity: A single plane-strain crack. Int. J. Eng. Sci., 88:73 - 82, 2015.

[50] J. L. Grenestedt, S. Hallström, and J. Kuttenkeuler. On cracks emanating from wedges in expanded PVC foam. Eng. Fract. Mech., 54(4):445-456, 1996.

[51] A. A. Griffith. The phenomena of rupture and flow in solids. Philos. T. R. Soc. S-A., 221:163-198, 1921.

[52] B. Halphen and Q. S. Nguyen. Sur les matériaux standards généralisés. J. Mécanique, 14(1):39-63, 1975.

[53] M. Z. Hossain, C.-J. Hsueh, B. Bourdin, and K. Bhattacharya. Effective toughness of heterogeneous media. J. Mech. Phys. Solids, 71:320-348, 2014.

[54] C. E. Inglis. Stresses in plates due to the presence of cracks and sharp corners. Transactions of the Institute of Naval Architects, 55:219-241, 1913.

[55] M. A. Issa, M. A. Issa, M. S. Islam, and A. Chudnovsky. Size effects in concrete fracture: Part I, experimental setup and observations. Int J. Fracture, 102(1):1-24, 2000.

[56] F. Iurlano. A density result for GSBD and its application to the approximation of brittle fracture energies. Calc. Var. Partial Dif., 51(1/2):315 - 342, 2014.

[57] A. Karma, D. A. Kessler, and H. Levine. Phase-field model of mode III dynamic fracture. Phys. Rev. Letters, 87(4), 2001. 
[58] M. Klinsmann, D. Rosato, M. Kamlah, and R. M. McMeeking. An assessment of the phase field formulation for crack growth. Comp. Meth. Appl. Mech. Engng., 294:313 - 330, 2015.

[59] C. Kuhn, A. Schlüter, and R. Müller. On degradation functions in phase field fracture models. Comp. Mater. Sci., 108, Part B:374-384, 102015.

[60] P. Lazzarin and S. Filippi. A generalized stress intensity factor to be applied to rounded v-shaped notches. Int. J. Solids Struct., 43(9):2461 - 2478, 2006.

[61] D. Leguillon. Strength or toughness? A criterion for crack onset at a notch. Euro. J. Mech. A/Solids, 21(1):61-72, 2002.

[62] D. Leguillon and E. Sanchez-Palencia. Computation of Singular Solutions in Elliptic Problems and Elasticity. John Wiley \& Sons, Inc., 1987.

[63] B. Li, C. Peco, D. Millán, I. Arias, and M. Arroyo. Phase-field modeling and simulation of fracture in brittle materials with strongly anisotropic surface energy. Int. J. Numer. Meth. Engng., 102(34):711-727, 2014.

[64] T. Li. Gradient Damage Modeling of Dynamic Brittle Fracture. PhD thesis, Université ParisSaclay - École Polytechnique, October 2016.

[65] T. Li, J.-J. Marigo, D. Guilbaud, and S. Potapov. Gradient damage modeling of brittle fracture in an explicit dynamics context. Int. J. Num. Meth. Engng., 52016.

[66] E. Lorentz and S. Andrieux. Analysis of non-local models through energetic formulations. Int. J. Solids Struct., 40:2905-2936, 2003.

[67] J.-J. Marigo, C. Maurini, and K. Pham. An overview of the modelling of fracture by gradient damage models. Meccanica, 51(12):3107-3128, 2016.

[68] C. Maurini, B. Bourdin, G. Gauthier, and V. Lazarus. Crack patterns obtained by unidirectional drying of a colloidal suspension in a capillary tube: experiments and numerical simulations using a two-dimensional variational approach. Int. J. Fracture, 184(1-2):75-91, 2013.

[69] A. Mesgarnejad, B. Bourdin, and M. M. Khonsari. Validation simulations for the variational approach to fracture. Comp. Methods Appl. Mech. Engng., 290:420-437, 2015.

[70] C. Miehe, F. Aldakheel, and A. Raina. Phase field modeling of ductile fracture at finite strains. a variational gradient-extended plasticity-damage theory. Int. J. Plasticity, 2016.

[71] C. Miehe, M. Hofacker, L.-M. Schänzel, and F Aldakheel. Phase field modeling of fracture in multi-physics problems. Part II. coupled brittle-to-ductile failure criteria and crack propagation in thermo-elastic-plastic solids. Comp. Meth. Appl. Mech. Engng., 294:486-522, 2015.

[72] A. Mielke. Evolution of rate-indipendent system. In C. Dafermos and E. Feireisl, editors, Handbook of Differential Equations, Evolutionary Equations, volume 2, chapter 6, pages 461-559. Elsevier, 2005.

[73] N. I. Muskhelishvili. Some Basic Problems of the Mathematical Theory of Elasticity: Fundamental Equations, Plane Theory of Elasticity, Torsion, and Bending (translated from Russian). Noordhoff International Publishing, Leyden, The Netherlands, 2nd edition, 1977.

[74] M. Negri and M. Paolini. Numerical minimization of the Mumford-Shah functional. Calcolo, 38(2):67-84, 2001.

[75] T. T. Nguyen, J. Yvonnet, M. Bornert, C. Chateau, K. Sab, R. Romani, and R. Le Roy. On the choice of parameters in the phase field method for simulating crack initiation with experimental validation. Int. J. Fracture, 197(2):213-226, 2016. 
[76] M. Ortiz and A. Pandolfi. Finite-deformation irreversible cohesive elements for three-dimensional crack-propagation analysis. Int. J. Num. Meth. Engng., 44:1267-1282, 1999.

[77] K. Pham, H. Amor, J.-J. Marigo, and C. Maurini. Gradient damage models and their use to approximate brittle fracture. Int. J. Damage Mech., 20(4, SI):618-652, 2011.

[78] K. Pham and J.-J. Marigo. Approche variationnelle de l'endommagement: I. Les concepts fondamentaux. The variational approach to damage: I. The foundations. C.R. Mécanique, 338(4):191$198,2010$.

[79] K. Pham and J.-J. Marigo. Approche variationnelle de l'endommagement: II. Les modèles à gradient. The variational approach to damage: II. The gradient damage models. C.R. Mécanique, 338(4):199-206, 2010.

[80] K. Pham, J.-J. Marigo, and C. Maurini. The issues of the uniqueness and the stability of the homogeneous response in uniaxial tests with gradient damage models. J. Mech. Phys. Solids, 59(6):1163 - 1190, 2011.

[81] K. H. Pham, K. Ravi-Chandar, and C. M. Landis. Experimental validation of a phase-field model for fracture. International Journal of Fracture, 205(1):83-101, 2017.

[82] J. R. Rice. The mechanics of earthquake rupture. In A. M. Dziewonski and E. Boschi, editors, Proc. Int. School of Physics "Enrico Fermi" Physics of the Earth's Interior, volume 555-649. North-Holland, 1980.

[83] J. M. Sargado, E. Keilegavlen, I. Berre, and J. M. Nordbotten. High-accuracy phase-field models for brittle fracture based on a new family of degradation functions. arXiv:1705.04046, 2017.

[84] B. Schmidt, F. Fraternali, and M. Ortiz. Eigenfracture: An eigendeformation approach to variational fracture. Multiscale Model. Simul., 7(3):1237-1266, 2009.

[85] A. Seweryn. Brittle fracture criterion for structures with sharp notches. Eng. Fract. Mech., 47(5):673-681, 1994.

[86] P. Sicsic and J.-J. Marigo. From gradient damage laws to Griffith's theory of crack propagation. J. Elasticity, 113(1):55-74, 2013.

[87] S. A. Silling. Reformulation of elasticity theory for discontinuities and long-range forces. J. Mech. Phys. Solids, 48(1):175-209, 2000.

[88] M. Strandberg. Fracture at V-notches with contained plasticity. Eng. Fract. Mech., 69(3):403-415, 2002.

[89] M. F. Wheeler, T. Wick, and W. Wollner. An augmented-lagrangian method for the phase-field approach for pressurized fractures. Comp. Meth. Appl. Mech. Engng., 271:69-85, 42014.

[90] Z. A. Wilson, M. J. Borden, and C. M. Landis. A phase-field model for fracture in piezoelectric ceramics. International Journal of Fracture, 183(2):135-153, 2013.

[91] Z. A. Wilson and C. M. Landis. Phase-field modeling of hydraulic fracture. J. Mech. Phys. Solids, 96:264 - 290, 2016.

[92] Z. Yosibash, A. Bussiba, and I. Gilad. Failure criteria for brittle elastic materials. Int. J. Fracture, 125(3-4):307-333, 2004.

[93] X. Zhang, C. Vignes, S. W. Sloan, and D. Sheng. Numerical evaluation of the phase-field model for brittle fracture with emphasis on the length scale. Comput. Mech., pages 1-16, 2017. 